\title{
Oral Sessions
}

\section{Diabetes mellitus 1}

March 27 (Sat.) 11:10-12:00

Room 11

0 1-1

Regulation of hepatic lipid metabolism by the transcription factor Stra13

Kazuhiro Nomura (1), Wataru Ogawa (1), Aki Emi (1), Masato Kasuga (2)

(1)Division of Diabetes, Metabolism and Endocrinology, Kobe University Graduate School of Medicin, Japan, (2)Research Institute, International Medical Center of Japan, Japan

To identify novel regulatory mechanisms of energy homeostasis in the liver, we analyzed the alterations of hepatic gene expression in various physiological and pathological conditions in mice with the use of Gene Chip analysis. We have found that hepatic expression of Stra13, a member of bHLH family of transcription factors, was stimulated by insulin both in vitro and in vivo. In cultured hepatocytes, overexpression of Stra13 stimulates the expression of SREBP1c, a master regulator of hepatic lipogenesis, and the reduction of Stra13 with the use of short hairpin RNA (shRNA) inhibits insulin-induced expression of SREBP1c, indicating that Stra13 contributes the regulation of the SREBP1c expression by insulin. In obese KKAy mice, the hepatic abundance of Stra13 as well as of SREBP1c is greater than those of control mice. The reduction of Stra13 in the liver with the use of an adenovirus vector encoding shRNA of Stra13 decreases the expression of SREBP1c along with those of the downstream genes of SREBP1c including stearoyl-CoA desaturase1 or fatty acid synthase. The reduction of hepatic Stra13 in KKAy mice markedly ameliorates hypertriglyceridemia and heyperinsulinemia. These results suggest that Stra13 controls hepatic lipid metabolism thorough regulating the expression of SREBP1c and that the Stra13 pathway serves as a potential therapeutic target of dyslipidemia and insulin resistance.

\section{1-2}

\section{Effect of alpha-1-antitrypsin on insulin resistance and type 2 diabetes}

Cheol Son (1,2), Hang Shi (1,3), Eleftheria Maratos-Flier (1), Jeffrry S. Flier (1)

(1)Division of Endocrinology, Beth Israel Deaconess Medical Center, Boston, MA, USA, (2)Department of Medicine and Clinical Science, Kyoto University Graduate School of Medicine, Kyoto, Japan, (3)Department of Internal Medicine, Wake Forest University, Winston-Salem, NC, USA

Alpha-1-antitrypsin (AAT) is a major serine protease inhibitor in circulation. Mutation of AAT that induces decrease of serum AAT concentration is known as the cause of pulmonary emphysema. Lack of anti-inflammatory effect of AAT has been considered as one possible mechanism causing this disease. Because of its anti-inflammatory effects, many researchers have studied about effects of AAT on type 1 diabetes. It is reported that treatment with AAT ablates invasive insulitis and restores euglycemia in NOD mice, a well-known model of type 1 diabetes. However, few studies have been published about the effect of AAT on type 2 diabetes. Because increasing data indicate that insulin resistance and type 2 diabetes are associated with activation of inflammatory pathways in metabolic tissues, we investigated the effects of AAT on glucose tolerance and insulin resistance in two animal models of type 2 diabetes, genetically obese $d b / d b$ mice and diet-induced obese mice (DIO mice). Method: Clinicalgrade human AAT or BSA (control purpose) was given to lean, $d b$ / $d b$ or DIO mice every 3 days. Glucose tolerance was examined by intraperitoneal GTT after the third injection. Insulin resistance was evaluated by ITT after the fourth injection. Results: AAT treatment improved GTT and ITT in both $d b / d b$ and DIO mice without changing body weight or fat mass. TNF- $\alpha$ gene expression was decreased in the adipose tissue of the $d b / d b$ and DIO mice compared with their BSA-injected control mice, suggesting that glucose tolerance and insulin resistance were improved by suppression of inflammation. Further studies are on-going.

\section{$01-3$}

Expression changes of phosphorylated Smad2 and Smad3 in the hippocampus of streptozotocin-induced diabetic cognitive impairment rats

\section{Yi Bao (1), Yongquan Shi (1), Junjie Zou (1), Ying Zhao (2), Zhimin Liu (1)}

(1)Department of Endocrinology, Changzheng Hospital, Second Military Medical University, Shanghai, China, (2)Department of Neurology, Changzheng Hospital, Second Military Medical University, Shanghai, China

Activation of the Smad signalling pathway has been implicated in the pathological process of diabetic associated complications. The current study was designed to see whether Smad signalling was activated in the hippocampus of streptozotocin-induced diabetic cognitive impairment rats. We employed streptozotocin (STZ)-induced diabetic rats and test their capacity for learning and memory by Morris water maze. We determined the expression level of phosphorylated Smad 2 and Smad 3 in the hippocampus by western blot analysis and double immunofluorescene labeling assay. 8 weeks after STZ induction, the diabetic rats showed decreased capacity for learning and memory. In diabetic rats the latency and distance swum reach to the platform increased significantly compared to controls $(\mathrm{P}<0.05)$, and the percentage of time spent in the quadrant of the pool with platform decreased notably $(\mathrm{P}<0.05)$.Compared with vehicle-treated controls, immunoblot analysis of hippocampal extracts showed that phosphorylated Smad 2 and Smad3 were upregulated at 8 weeks post streptozotocin induction $(\mathrm{P}<0.01)$. In addition, immunofluorescence labelling assay showed that the percentage of pSmad $2 \mathrm{immu-}$ noreactive astrocytes increased significantly in CA1, CA3 and dentate gyrus region $(\mathrm{P}<0.01)$, and $\mathrm{pSmad} 3$ immunoreactive astrocytes increased significantly in $\mathrm{CA} 1$ region $(\mathrm{P}<0.01)$ and in $\mathrm{CA} 3$ and dentate gyrus region $(\mathrm{P}<0.05)$ of the hippocampus in diabetic rats. These data indicate that Smad signalling is enhanced in hippocampal astrocytes of diabetic rats, and may thereby represent a clue to explore its exact role in the development of diabetic encephalopathy.

\section{$01-4$}

\section{Insulin signaling via PKC $\delta$ isoforms in the diabetic brain promotes cognitive function}

Niketa A. Patel (1), Denise R. Cooper (1), Andre Apostolatos (1)

(1)JAHVA and Univ South Florida, Tampa, FL, USA

People with type II diabetes have a significantly higher rate of decline in cognitive function. However, the mechanisms are poorly understood. There are strong links between insulin and cognitive function supported by epidemiological data from humans and animal studies and in vitro research. Protein kinase $\mathrm{C}$ (PKC) signaling cascades and insulin pathways are closely integrated. The consequences of PKC activation by insulin in the CNS influence memory, cognition, synaptogenesis, and neuronal repair. In addition, $\mathrm{PKC} \delta$, a novel PKC has been implicated in memory, neuronal survival and proliferation. Insulin regulates the alternative splicing of mouse $\mathrm{PKC} \delta$ splice vari-

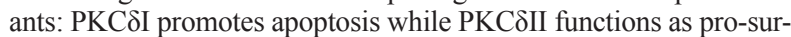


vival protein. In vitro and in vivo studies demonstrated that apoptosis accounted for the neuronal loss and cognitive decline in the diabetic mouse models. Our data in mouse neural cells derived from the hippocampus, which is the seat of learning, memory and cognition, demonstrates that insulin increases the expression of PKCסII. PKC $\delta I$ expression is not influenced by high glucose or thiazolidinediones thereby establishing that insulin increases PKC $\delta I$ expression via its signaling pathways To determine which pathways are involved in insulin-mediated $\mathrm{PKC} \delta$ alternative splicing in neuronal cells, we used inhibitors of several pathways targeted by insulin in other tissues and show that insulin increases PKCסII expression via PI3K-Akt pathway. Constitutively active Akt2 kinase mimicked insulin effects in increasing PKC $\delta$ II levels while WT-Akt2 kinase had no effect without insulin. Further, we show that PKC $\delta I$ increases expression of pro-survival proteins $\mathrm{Bcl}-2$ and $\mathrm{Bcl}-\mathrm{xL}$ thereby increasing neurogenesis.

\section{Diabetes mellitus 2}

March 27 (Sat.) 11:10-12:00

Room 12

O 2-1

HNGF6A, a novel, non-IGFBP-3 binding, humanin analog, with enhanced biological activity, superior pharmacokinetic profile, and no perturbation of the IGF system

Jyotsna Keni (1), Hemal H Mehta (1), David Hwang (1), Paulette M Yamada (1), Haruo Mizuno (1), Kenichi Miyako (1), Laura J Cobb (1), Pinchas Cohen (1)

(1)UCLA Mattel Children's Hospital, University of California, Los Angeles, CA, USA

BACKGROUND: Humanin is a mitochondrially-derived peptide, with potent neurosurvival and insulin-sensitizing effects. Humanin binds with high-affinity to IGFBP-3, which modulates the activity of both humanin and IGF-I. HNGF6A is novel humanin-analog with loss of IGFBP-3-binding and enhanced neurosurvival-activity. OBJECTIVES: 1)To utilize a newly-developed humanin ELISA to establish normative-ranges of humanin in mice, 2)To establish the effects of circulating IGFBP-3 on humanin-pharmacokinetics, and 3) To compare the pharmacokinetics of HNG and HNGF6A. RESULTS: Plasma was collected from C57/BL6 mice of both genders at varying ages and analyzed by in-house novel humanin ELISA. Humanin-levels are lower in females and decline with age in mice of both genders. 11-week-old male mice given intra-peritoneal injections of 2-mg/kg HNG or HNGF6A; and EDTA plasma was collected at multiple timepoints (0-180-minutes). Baseline levels of humanin are 3-fold lower in IGFBP3KO mice compared with wildtype-littermates. Humanin levels peaked at 10-minutes in both strains, but IGFBP3KO-mice displayed a longer humanin half-life compared to wildtype-mice. When HNGF6A was injected into wildtype-mice, the peak was similar to HNG, but the half-life was 3-fold longer. Exogenous-humanin led to a $30 \%$ decrease in endogenousIGFBP-3 and IGF-1-levels, however HNGF6A had no effect on IGF/IGFBP-3 physiology. SUMMARY: HNGF6A is a highly potent humanin analog with a longer half-life, which does not disturb the IGF system and may contribute to longer lasting humanin-based therapeutics for the treatment and prevention of diabetes and neurodegenerative diseases.

\section{2-2}

\section{Improved metabolic parameters in diabetes patients after} joining diabetes shared care program in Taiwan

Jiang Ju Ying (1), Hua Fen Chen (1), Mei Hsiu Chen (1), Ling Yuh Hung (1), Pi Lien Hung (1), Hsin Yu Lee (1), Ming Tsang Lee (1), Ting Ting See (1), Chih-Yuan Wang (1)

(1)Division of Endocrinology and Metabolism, Department of Internal Medicine, Far Eastern Memorial Hospital, Taipei county, Taiwan

Objective : Comprehensive diabetes education program is important in order to achieve glycemic control target to avoid chronic complications in diabetes. Hence, Taiwan government implemented diabetes shared care program since 2001 . We aimed to compare the change in metabolic parameters before and after diabetes subjects joining the program. Methods and Materials : We enrolled 2,166 diabetes patients into this program between 2002 and 2008. They could receive comprehensive diabetes education including diet and exercise consultation at least four times per year from certified diabetes educator and dietitian. The study outcome included mean change in fasting plasma glucose, glycated hemoglobin (Hbalc), total cholesterol, low-density lipoprotein cholesterol (LDL-C), high-density lipoprotein cholesterol (HDL-C) and triglycerides (TG). All these data were collected retrospectively and analyzed with SPSS 16.0 software including descriptive statistic analysis and paired $t$ test. Results : A total of 1,453 patients complete follow up for at least one year. Mean age of patients was $56.80 \pm 12.71$ years and duration of diabetes was $4.98 \pm 5.86$ years. Significant reduction $(\mathrm{P}<0.001)$ in fasting plasma glucose $(180.91 \pm 81.10 \mathrm{mg} / \mathrm{dL}$ to $152.52 \pm 57.36 \mathrm{mg} / \mathrm{dL})$, Hbalc $(9.46 \pm 2.26 \%$ to $8.00 \pm 2.75 \%)$, triglyceride $(191.66 \pm 218.27 \mathrm{mg} /$ $\mathrm{dL}$ to $173.87 \pm 158.67 \mathrm{mg} / \mathrm{dL})$, total cholesterol $(198.49 \pm 50.46 \mathrm{mg} /$ $\mathrm{dL}$ to $187.37 \pm 38.93 \mathrm{mg} / \mathrm{dL})$ and LDL-C $(115.52 \pm 37.30 \mathrm{mg} / \mathrm{dL}$ to $111.43 \pm 33.33 \mathrm{mg} / \mathrm{dL}$ ), no significant difference was found in HDL-C level. Conclusion : Implementation of diabetes shared care program enable comprehensive diabetes education being delivered to diabetes subjects and our patients successfully achieve glycemic and dyslipidemia control target after joining the program.

\section{2-3}

Effects of variations in retinol binding protein 4 gene and adipose specific expression of gestational diabetes in Beijing China

Fan Ping (1), Hong-ding Xiang (1), Ming Li (1), Wei Li (1), Jun Liu (2), Min Nie (1), Yuan-chi Hui (1)

(1)Key Laboratory of Endocrinology, Ministry of Health,Department of Endocrinology, Peking Union Medical College Hospital, Peking Union Medical College, Chinese Academy of Medical Sciences, China, (2) Department of Obstetrics \& Gynecology, Peking Union Medical College Hospital, Peking Union Medical College, Chinese Academy of Medical Sciences, China

Objective: To investigate the clinical features of gestational diabetes mellitus (GDM) in China and the effects of RBP4 genetic variants, and also to identifyRBP4 expression changes at mRNA and protein levels. Research design and methods: 1595 Chinese pregnant women were included in the study. Four known RBP4 single nucleotide polymorphisms (SNPs) were genotyped in 505 cases and 687 controls. Serum RBP4 level was measured in 74 cases and 69 controls. The levels of expression of adipose specific RBP4 mRNA and protein were detected in 41 samples of subcutaneous adipose tissue. Results: The estimated indices of insulin resistance were significantly increased in GDM while $\beta$-cell functions were decreased. Two single SNPs were associated with GDM in case-control study (rs3758539 G vs A, $\mathrm{OR}=1.446,95 \%$ CI 1.095-1.911, $\mathrm{P}=0.009$; rs $3758539 \mathrm{GG}$ vs AG+AA, $\mathrm{OR}=1.532,95 \% \mathrm{CI} 1.131-2.075 ; \mathrm{rs} 12265684 \mathrm{G}$ vs C, OR $=1.296,95 \% \mathrm{CI}$ 1.014-1.658). Furthermore, a haplotype of 3 common SNPs [G-G-T] was significantly increased in cases compared with controls $(\mathrm{P}=0.016$, $\mathrm{OR}=1.322,95 \%$ CI 1.054-1.659). RBP4 mRNA expression in adipose tissue also significantly increased in GDM compared with the controls. The levels of serum RBP4 and adipose specific protein were somewhat higher than the controls; however, there were no statistical differences. Conclusions: The variants of RBP4 gene appeared to be involved in the development of GDM in Beijing China. On adipose-specific mRNA level, we found RBP4 expression increased in GDM. Concerning RBP4 protein level, no statistical differences were observed either in serum or in adipose tissue.

O 2-4

Oral magnesium supplementation can improve glycemic control and blood pressure in type 2 diabetic patients

Elham Ouspid (1), Saeedeh Hosseini (1), Mehrdad Solati (1), Nepton Soltani (2), Mansoor Keshavarz (3)

(1)Department of Internal Medicine and Endocrinology, Bandar Abbas Faculty of Medicine, Hormozgan University of Medical Sciences, Iran, (2)Physiology Department, Bandar Abbas Faculty of Medicine, Hormozgan University of Medical Sciences, Iran, (3)Physiology Department, School of Medicine, Tehran University of Medical Sciences, Tehran, Iran

OBGECTIVE: Magnesium is the second most abundant intracellular cation. $\mathrm{Mg}$ homeostasis and metabolism is affected by multiple factors but it is in part, a determinant of cellular ionic environment 
and cellular responsiveness to external stimuli such as insulin and glucose. It plays an important role in insulin homeostasis and glucose metabolism through multiple enzymatic reactions. In addition $\mathrm{Mg}$ can affect vascular smooth muscle tone and contractility, and as a result blood pressure. So with increasing data on magnesium deficiency in diabetic patients and epidemiological studies demonstrating magnesium deficiency as a risk factor for diabetes, it is logical to search for its possible beneficial effects on diabetes control and prevention. Our purpose was to determine whether oral magnesium supplementation improves metabolic control, lipid profile, blood pressure, hepatic enzymes, hemoglobin concentration and anthropometric indices in type 2 diabetic patients. RESEARCH DESIGN AND METHODS: 42 diabetic patients were included in a Randomized Double Blind Placebo-Controlled Clinical Trial, and received either placebo or $300 \mathrm{mg}$ elemental magnesium (as MgSo4) daily, for 3 months. Parameters of metabolic control, lipid profile, blood pressure, magnesium status, hepatic enzymes, hemoglobin concentration and anthropometric indices were determined at the beginning and the end of the study. RESULTS: Daily administration of $300 \mathrm{mg}$ elemental magnesium, for 3 months, caused statistically significant improvement of fasting blood glucose, $2 \mathrm{hr}$ post parandial glucose, lipid profile, blood pressure and hepatic enzymes. CONCLUSION: Chronic oral magnesium supplementation with proper dosage, have beneficial effects on metabolic control and complications of type 2 diabetes.

\section{Thyroid 1}

March 27 (Sat.) 11:10-12:00

Room 13

0 3-1

Liganded-thyroid hormone receptor- $\alpha$ induces proliferation of pancreatic $\beta$-cells

Fumihiko Furuya (1), Hiroki Shimura (1), Toyoshi Endo (1), Tetsuro Kobayashi (1) (1)The Third Department of Internal Medicine, University of Yamanashi, Yamanashi, Japan

Failure of the functional pancreatic $\beta$-cell mass to expand in response to increased metabolic demand is a hallmark of type 2 diabetes. Lineage tracing studies indicate that replication of existing $\beta$-cells is the principle mechanism of $\beta$-cell proliferation in adult animals. In the rat pancreas, we found that thyroid hormone receptor $\alpha(\mathrm{TR} \alpha)$ mRNA shows 58.6-fold greater expression at the end of embryogenesis compared with the level in adults. This suggests that TR $\alpha$ is required for $\beta$-cell proliferation or replication. To identify the rule of TR $\alpha$ in the processes of $\beta$-cell growth and cell cycle regulation, we constructed two recombinant adenovirus vectors, which were AdTR $\alpha$ and Ad-TR $\alpha$ PV (a dominant negative mutant). Infection with AdTR $\alpha$ increased the expression of cyclin D1 mRNA and protein, along with cell cycle progression and cell proliferation, in the presence of tri-iodothyronine (T3). In contrast, this T3-dependent induction of cyclin D1 and cell cycle progression was not observed in AdTR $\alpha$ PV-infected cells. Furthermore, intrapancreatic injection of AdTR $\alpha$ led to the restoration of islet function and an increase of the $\beta$-cell mass in immunodeficient mice with streptozotocin-induced diabetes. These results support the hypothesis that ligandedTR $\alpha$ plays a critical role in $\beta$-cell replication and expansion of the $\beta$-cell mass during postnatal development. Thus, liganded-TR $\alpha$ may be a target for therapeutic strategies that can induce the expansion and regeneration of $\beta$-cells.
0 3-2

Thyrotropin-releasing hormone affects gene expression in the islet of Langerhans of the pancreas

Garay Jennifer (1), Nobuyuki Shibusawa (1), Kazuhiko Horiguchi (1), Yasuyo Nakajima (1), Emi Ishida (1), Koshi Hashimoto (1), Tetsurou Satoh (1), Masanobu Yamada (1), Masatomo Mori (1)

(1)Department of Medicine and Molecular Science, Gunma University Graduate School of Medicina, Gunma, Japan

Thyrotropin-releasing hormone (TRH), a hypothalamic tripeptide, plays a key role in the regulation of the thyroid axis. In addition to the hypothalamus, TRH has been identified in many systemic organs including the pancreas. The TRH-deficient mice $\left(\mathrm{TRH}^{--}\right)$ showed characteristic tertiary hypothyroidism and also developed hyperglycemia, which was accompanied by impaired insulin secretion in response to glucose. To elucidate the molecular mechanism of TRH effect in the pancreas, we conducted a microarray study with islets of Langerhans isolated from $\mathrm{TRH}^{-/}$mice pancreas. In the result of microarray analysis, we focused fibroblast growth factor 21 (FGF21), one of genes down regulated with $\mathrm{TRH}^{--}$. FGF21 plays an important role in the regulation of glucose, lipid, and energy homeostasis. It has been shown to preserve $\beta$-cell mass and restore $\beta$-cell function in the pancreas. The FGF21 mRNA expression levels in islets of $\mathrm{TRH}^{-/-}$were $40-60 \%$ less compared with those of $\mathrm{TRH}^{+/+}$ mice, which could not be reversed by thyroid hormone treatment. In transfection repoter assay, TRH stimulated human FGF21 promoter activity in the $\mathrm{CHO}$ cells that expressed TRH receptor.These results suggested that FGF21 could be one of key factors of insulin secretion in the pancreas and regulation of blood glucose level associated with TRH.

0 3-3

Effects of selenium on $\mathrm{CD4}^{+} \mathrm{CD}^{2} 5^{+}$regulatory T cells in NOD.H$2^{\text {h4 }}$ mice with spontaneous autoimmune thyroiditis

Haibo Xue (1), Zhongyan Shan (1), Weiwei Wang (1), Weiping Teng (1)

(1)Department of Endocrinology and Metabolism, the First Affiliated Hospital, China Medical University, Shenyang, P.R. China

Objects Selenium (Se) is essential for thyroid hormone synthesis and metabolism. Recent clinical studies have demonstrated that Se treatment reduced serum thyroid-specific antibody titers in patients with autoimmune thyroiditis (AIT), but the mechanism is unclear. The aim of our study was to investigate the effects of Se treatment on $\mathrm{CD} 4{ }^{+} \mathrm{CD} 25^{+}$regulatory $\mathrm{T}$ cells (Treg) in spontaneous autoimmune thyroiditis (SAT) model. Methods NOD.H-2 ${ }^{\text {h4 }}$ mice were randomly divided into control and Se treatment groups. Mice were fed with $0.005 \% \mathrm{NaI}$ water for 8 weeks to induce SAT, and then Se-treated mice received $0.3 \mathrm{mg} / \mathrm{L}$ sodium selenite in drinking water. We measured thyroid weight, serum thyroglobulin antibody (TgAb), lymphocytic infiltration in thyroids, serum selenium, percentage of CD $4{ }^{+} \mathrm{CD} 25^{+} \mathrm{Foxp}^{+} \mathrm{T}$ cells and Foxp3 mRNA expression in splenocytes. Results The SAT mice had fewer Treg cells and reduced Foxp3 mRNA expression in splenocytes compared with the controls $(P<0.01)$. The percentage of $\mathrm{CD} 4^{+} \mathrm{CD} 25^{+} \mathrm{Foxp} 3^{+} \mathrm{T}$ cells and expression of Foxp3 mRNA were increased by Se treatment (as compared with untreated SAT mice, $P<0.05$ ). Mice that received Se supplementation also had lower serum $\mathrm{TgAb}$ titers and reduced lymphocytic infiltration in thyroids than untreated SAT mice. Conclusion Treg cells may play an important role in the development of SAT and Se treatment leads to the suppression of SAT by restoring Treg cells by up-regulating the expression of Foxp3 mRNA.

\section{3-4}

\section{Identification of microRNAs that regulate thyroid physiology}

Takeshi Akama (1), Akira Kawashima (1), Huhehasi Wu (1), Kazunari Tanigawa (1), Mariko Sue (1), Yuko Ishido (1), Koichi Suzuki (1)

(1)Laboratory of Molecular Diagnostics, Department of Mycobacteriology, National Institute of Infectious Diseases, Tokyo, Japan

Endogenous short RNAs, known as microRNAs (miRNAs) regulate expression of their target genes. To date 10,883 miRNAs including 721 for human are identified. However, most of their function 
as well as the target genes are yet to be determined, although some of them are thought to be involved in the regulation of cell cycle or apoptosis. The role of miRNA on endocrine functions, especially those on the physiological function of the thyroid is not known.

In order to explore possible function of miRNA in the thyroid physiology, we have compared their expression levels using rat thyroid FRTL-5 cells under stimulation with thyrotropin (TSH) and thyroglobulin (Tg). After stimulation, cellular RNA was extracted and the expression of miRNA was determined using DNA microarray analysis. Expression of 5 miRNAs was significantly suppressed by TSH, 18 were downregulated by Tg and 4 miRNAs were modulated both by TSH and Tg. The expression levels of these miRNAs were confirmed by real-time PCR analysis. Then the role of these miRNAs was evaluated by transfecting miRNA agonist (pre-miR) or antagonist (anti-miR) to express and suppress specific miRNA in FRTL-5 cells, respectively. We have identified some miRNA that seems to mediate $\mathrm{Tg}$ action to regulate cell proliferation. Our results indicated that miRNAs are involved in the regulation of physiological function of the thyroid. These would be a novel therapeutic target to treat thyroid diseases.

\section{Bone and mineral metabolism}

March 27 (Sat.) 11:10-12:00

Room 14

\section{4-1}

\section{PTH inhibition rates are useful in the detection of early-stage} primary hyperparathyroidism

Lin Zhao (1), Min jia Zhang (1), Hong-yan Zhao (1), Li-hao Sun (1), Jie-li Li (1), Bei Tao (1), Weiqing Wang (1), Guang Ning (1), Jian-min Liu (1)

(1)Shanghai Institute of Endocrine and Metabolic Diseases, Rui Jin Hospital, Shanghai Jiaotong University School of Medicine, China

An increasing number of individuals display mildly elevated serum calcium concentrations or normocalcemia with elevated serum PTH levels, and some of them are surgically confirmed as primary hyperparathyroidism. Simple and practical methods of diagnosing early stage PHPT are lacking, especially when the situation is complicated by the presence of vitamin $\mathrm{D}$ deficiency. The objective of this study was to establish biochemical thresholds for the intravenous calcium suppression test in the early diagnosis of PHPT.This study involving 133 patients with elevated serum PTH concentrations and 20 normal controls. Patients were divided into three groups: Group 1: surgically proven hypercalcemic PHPT (serum Ca $>2.75 \mathrm{mmol} / \mathrm{l}$ ), Group 2:surgically proven mild PHPT (serum Ca:2.15-2.75mmol/1), and Group 3: normocalcemia with elevated serum PTH, but the diagnosis of PHPT has not been determined. Calcium suppression tests were performed in Groups 2, 3 and the controls. The serum PTH inhibition rate was less pronounced in Group 2 compared with Group 3 $(\mathrm{P}<0.001)$ and the controls $(\mathrm{P}<0.001)$. Receiver operating characteristic curve analysis suggests that a serum calcium level higher than $2.43 \mathrm{mmol} / 1$ and a PTH-IR less than $73 \%$ in the calcium suppression test may differentiate Group 2 from normal controls. The mean serum $25(\mathrm{OH}) \mathrm{D}$ levels were $<50 \mathrm{nmol} / \mathrm{l}$ in all groups, with the lowest values observed in Group 1.It is quite useful to combine serum calcium levels with the PTH-IR to identify patients with early stage PHPT, especially in the presence of vitamin D deficiency.

0 4-2

BA058, a novel analog of human parathyroid hormone-related peptide (PTHrP), induces a dose-dependent increase in BMD without the dose-limiting hypercalcemia of teriparatide: Results of a randomized, placebo-controlled phase 2 study

Louis St.L. O'Dea (1), Gary Hattersley (1), C. Richard Lyttle (1)

(1)Radius Health Inc," Cambridge, Massachusetts, USA

BA058 is a synthetic 34 amino acid analog of hPTHrP designed for less resorptive and hypercalcemic effect and greater anabolic effect than hPTH. A Phase 2 study at 40 study centers in the USA, UK, Argentina and India treated 221 patients, randomized to BA058 pla- cebo, $20 \mathrm{mcg}$, $40 \mathrm{mcg}, 80 \mathrm{mcg}$, or teriparatide (FORTEO; Eli Lilly) $\mathrm{SC}$ daily. Mean patient age was 69 and mean spinal BMD T-score was -2.9. Following 6 months of treatment, mean changes in spinal BMD were $1.4 \%, 3.5 \%, 4.9 \%, 6.7 \%$ and $6.0 \%$ for placebo, BA058 $20 \mathrm{mcg}, 40 \mathrm{mcg}, 80 \mathrm{mcg}$ and teriparitide, respectively. The linear trend test across dose was significant $(\mathrm{p}<0.001)$ and all BA058 dose groups were different from placebo $(\mathrm{p}=0.022,<0.001$ and $<0.001$, respectively), as was teriparatide $(\mathrm{p}<0.001)$. The corresponding change in total hip BMD was $0.2 \%, 1.3 \%, 1.5 \%, 2.9 \%$ and $0.6 \%$, respectively. Anabolic bone markers also showed a dose-dependent linear statistically significant rise over treatment (PINP, Osteocalcin and bone-specific Alkaline Phosphatase).Hypercalcemia (>10.5 mg/ dL) occurred in $4 \%, 12 \%, 19 \%, 18 \%$, and $40 \%$ in the BA058 placebo, $20 \mathrm{mcg}$, $40 \mathrm{mcg}, 80 \mathrm{mcg}$, and teriparatide groups, respectively. BA058 was well tolerated. There were no treatment-related serious adverse events. After 12 months, mean changes in spinal BMD in a subset of continuing patients were $0.7 \%, 5.1 \%, 9.8 \% 12.9 \%$ and $8.6 \%$ for placebo, BA058 $20 \mathrm{mcg}, 40 \mathrm{mcg}, 80 \mathrm{mcg}$ and teriparitide, respectively, and $0.7 \%, 2.0 \%, 2.1 \%, 2.7 \%$ and $1.3 \%$, respectively, for total hip BMD.BA058 demonstrates greater gain in spine and hip BMD with lower rates of hypercalcemia than teriparatide, and is well tolerated.

0 4-3

\section{The endoplasmic reticulum-associated protein, OS-9, specifically recognizes immature processed forms of the calcium-sensing receptor}

Bryan K. Ward (1,2), Aaron L. Magno (1,2), Ajanthy Arulpragasam (1,2), Shelby Chew Wan Cher (1,2), Bernadette T. Pedersen (1,2), Evan Ingley (2), Arthur D. Conigrave (3), Thomas Ratajczak $(1,2)$

(1)Department of Endocrinology and Diabetes, Sir Charles Gairdner Hospital, Nedlands, Western Australia, Australia, (2)Centre for Medical Research, University of Western Australia, Nedlands, Western Australia, Australia, (3)School of Molecular and Microbial Biosciences, University of Sydney, New South Wales, Australia

The calcium-sensing receptor $(\mathrm{CaR})$ is a Class $\mathrm{C}$, $\mathrm{G}$ protein-coupled receptor which undergoes post-translational modification, including glycosylation, in the endoplasmic reticulum (ER). OS-9 is an ER-located lectin which has a quality-control function, selectively binding aberrantly folded glycoproteins for targeted, proteasomal destruction via the ER-associated degradation (ERAD) pathway. This selectivity is mediated by the N-terminally-located mannose 6-phosphate receptor homology (MRH) domain of OS-9 which can recognize specific glycans arising after mannose trimming of improperly folded proteins. We performed a yeast two-hybrid screen of a haemopoietic cell line library using the $\mathrm{CaR}$ intracellular tail as bait and identified OS-9 as a CaR-interacting protein. Yeast two-hybrid deletion mapping delineated the binding region to a C-terminal domain of OS- 9 and to amino acids $948-980$ of the CaR tail. Confocal microsopic analysis of EGFP-tagged OS-9 and FLAG-tagged CaR co-expressed in COS- 1 cells demonstrated colocalization of OS-9 and the $\mathrm{CaR}$ in the ER. Moreover, immunoprecipitation studies using lysates from COS-1 cells co-expressing S-tagged OS-9 and FLAG-tagged CaR revealed that OS-9 targets the CaR, but specifically only the immature, high mannose form of the CaR, not the fully glycosylated, mature form found on the cell membrane. These results suggest that OS-9 targets immature, misfolded $\mathrm{CaR}$ for destruction through the ERAD pathway and hints at dual recognition of CaR by OS-9; C-terminal recognition being protein-type specific and N-terminal recognition involved in determining aberrant folding via the MRH domain. Altered OS-9 recognition might therefore have major implications for $\mathrm{CaR}$ expression and disease syndromes in which the $\mathrm{CaR}$ is pivotal. 
0 4-4

\section{Glucuronized steroids inhibited a-Klotho-dependent FGF23} signaling-therapeutic implications for FGF23-caused rickets-

Ryota Maeda (1), Tomohiro Tanaka (1), Akihiro Imura (1), Yo-ichi Nabeshima (1,2)

(1)Department of Pathology and Tumor Biology, Graduate School of Medicine, Kyoto University, Kyoto, Japan, (2)Core Research for Evolutional Science and Technology, Japan Science and Technology Corporation, Saitama, Japan

Recently we have identified that $\alpha$-Klotho functions as a key regulator of the ionic calcium and phosphate homeostasis. Indeed, mice without expression of $\alpha$-Klotho died before adolescent due to the ionic disorder. We have reported that $\alpha$-Klotho functions to recruit $\mathrm{NaK}$-ATPase for rapid transport of calcium into body fluid and for a responsive secretion of parathyroid hormone $(\mathrm{PTH})$, and turns the FGF receptor (FGFR) as a canonical receptor for FGF23, which consequently reduces vitamin D synthesis and active transport of phosphate in kidney.

Patients with overdosed FGF23 have been suffered from rickets (low phosphate concentration in fluid along with decreased vitamin D synthesis). Neutralizing antibodies for FGF23 have been established to cure the patients with the FGF23-dependent rickets.

Here we demonstrated that physiological activity of FGF23 is completely depended on the $\alpha$-Klotho expression in vivo. Together with the data of which a mutated FGF23 that could not bind to $\alpha$-Klotho failed to activate FGFR, inhibition of the binding of FGF23 and $\alpha$-Klotho would rescue the patients of rickets with overactivated FGF23 signaling.

We have identified that Estrone-3- $\beta$-D-glucuronide (EstroneGlcA), a glucuronized steroid, specifically bound to $\alpha$-Klotho and inhibited its enzymatic activity. Here we show that Estrone-GlcA inhibited the $\alpha$-Klotho dependent FGF receptor activation as well. Estrone-GlcA competitively bound to $\alpha$-Klotho with FGF23/ FGFR and consequently impedes to form an active complex of FGF23 and FGFR. Now we propose that the glucuronized stroid would be a lead compound of chemical drugs for patients of FGF23and $\alpha$-Klotho-dependent rickets.

\section{Pituitary / Neuroendocrinology 1}

March 27 (Sat.) 11:10-12:00

Room 16

0 5-1

Infundibulo-hypophysitis associated with IgG4-related systemic disease: Is it primary or secondary?

Akira Shimatsu (1), Ichiro Fujisawa (2), Toshiaki Sano (3), Satoko Shimazu (1), Kazutaka Nanba (1), Hidenori Nomura (1), Tamiko Tamanaha (1), Tetsuya Tagami (1), Takeshi Usui (1), Mitsuhide Naruse (1)

(1)Clinical research Institute, National Hospital Organization Kyoto Medical Center, Kyoto, Japan, (2) Department of Radiology, Kishiwada City Hospital, Kishiwada, Japan, (3)Department of Pathology, Institute of Health Biosciences, The University of Tokushima Graduate School of Medicine, Tokushima, Japan

Inflammatory lesions of the pituitary gland and stalk are rarely encountered. Recently, the concept of IgG4-related sclerotic disease was proposed from Japan, and more than 20 cases have been reported as possibly associated with infundibulo-hypophysitis since 2000. We have reviewed such case reports in the published literature and in the abstracts of scientific meetings.

Twenty-five cases (male 21, female 3, unknown 1, age ranged from 44 to 77, median: 64 yrs) have been reported. Presenting symptoms were general fatigue $(n=12)$, headache(8), visual disturbances(6), polyuria(6) and fever(5). Various degrees of anterior pituitary hormone deficiencies(21) and diabetes insipidus(13) were demonstrated. Magnetic resonance imaging showed thickened pituitary stalk and/or pituitary mass. Pachymeningitis(6), parasinusitis(4) and orbital lesion(2) were noted. These structures shrank remarkably in response to steroid therapy, even in the lower dose range similar to that prescribed as replacement for adrenocortical insufficiency. Some of the anterior pituitary insufficiencies may also be resolved by steroid administration. Associated IgG4-related systemic diseases included involvement of lung(10), retroperitoneal fibrosis(9), Mikulicz disease/salivary gland(8), pancreas(7) and lymph nodes(4). Elevated levels of serum IgG4 were demonstrated in 12 cases. Pituitary pathology was investigated in 7 cases demonstrating the inflammatory pseudotumor with fibrosis and infiltration of IgG4-positive inflammatory cells.

Autoimmunity is not established as the pathogenesis for IgG4related systemic disease. The presence of pachymeningitis and parasinusitis suggested that sellar and parasellar structures were involved in chronic inflammation. We, therefore, classify this disorder not as a variant form of primary autoimmune hypophysitis but as secondary infundibulo-hypophysitis associated with IgG4-related systemic disease.

\section{5-2}

\section{Multiple dosing of a pegylated long-acting recombinant human growth hormone in adults subjects with growth hormone deficiency}

Michael Hojby Rasmussen (1), Birgit Sehested Hansen (1), Marianne Klose Klose (2), Mette Hansen (3), Esben Sondergaard (4), Marianne Andersen (3),

Ulla Feldt-Rasmussen (2), Torben Laursen (4), Jesper Madsen (1)

Jens S. Christiansen (4)

(1)Novo Nordisk A/S, Bagsvaerd, Denmark, (2)National University of Copenhagen, Denmark, (3)Odense University Hospital, Denmark, (4)Aarhus University Hospital, Denmark

Recombinant human Growth Hormone (rhGH) is normally administered as daily subcutaneous injections. NNC126-0083 is a pegylated rhGH developed with the aim of a once weekly hGH treatment.

The objectives of this study were to evaluate the safety, tolerability, pharmacokinetics and pharmacodynamics of multiple doses of NNC126-0083 in adult subjects with growth hormone deficiency (AGHD).

Subjects diagnosed with AGHD, on GH replacement therapy, age 20-65 years, BMI of $18.5-35.0 \mathrm{~kg} / \mathrm{m}^{2}, \mathrm{HbA} 1 \mathrm{C}>8.0 \%$ and not on insulin treatment were included. Fourteen days before randomisation the subjects discontinued their GH replacement therapy.

Four groups of 8 subjects were dosed once weekly for three weeks with a subcutaneous administra-tion of NNC126-0083 $(n=6)$ or placebo $(\mathrm{n}=2)$. The doses were escalated between cohorts in a sequential mode; from 0.01 to $0.08 \mathrm{mg}$ protein $/ \mathrm{kg} / \mathrm{week}$. Blood samples for assessment of safety, pharmacokinetics and pharmacodynamics (insulin-like growth factor-I (IGF-I)) were sampled up to 168 hours after first dosing and up to 240 hours after third dosing.

Results showed dose-proportional pharmacokinetics for the three lowest doses of NNC126-0083. At the highest dose a deviation from dose-proportionality was seen. No accumulation was observed. NNC126-0083 increased IGF-I dose-dependently. Steady state appeared to have been reached at second dose. The compound was well-tolerated with no significant difference in local tolerability compared to placebo. No antibody formation was recorded. The results indicate that $\mathrm{NNC126-0083} \mathrm{has} \mathrm{the} \mathrm{potential} \mathrm{as} \mathrm{an} \mathrm{effica-}$ cious, safe and well-tolerated once-weekly treatment for growth hormone deficiency in children and adults.

\section{5-3}

Short-term effects of weekly based slow releasing growth hormone (GH) on abdominal obesity patients

Sung-Woon Kim (1), Byung-Joon Kim (2), Jung-Hyun Noh (3), Mung-A Jung (4) Hyun-Ha Chang (5), Tae-Gwan Park (6)

(1)Department of Endocrinology, College of Medicine, Kyung Hee University, Seoul, Republic of Korea, (2) Endocrinology, Geonyang University, Daejeon, Republic of Korea, (3)Endocrinology, Baik Hospital, Inje University, Kyunggi-do, Republic of Korea, (4)Nephrology, Korea Cancer Center Hospital, Seoul, Republic of Korea, (5)Infection, Kyungbook National University, Daegu, Republic of Korea, (6)Ophthalmology, Soonchunhyang University, Gyunggi-do, Republic of Korea

Recently, we finally could use domestically developed once weekly regimen of rhGH. We enrolled 40 central obese patients from 2007.3 to 2008.4. The patients were selected with abdominal girth, as $>90$ $\mathrm{cm}$ for males, and $>85 \mathrm{~cm}$ for females and treated with weekly based rhGH for six months. Male to female ratio was $6 / 21$. Mean age was $52 \pm 8$ years. All patients were injected $2 \mathrm{mg}$ weekly for six months, and then analyzed body fat by DEXA, visceral fat by CT before and after treatment. The gender differences were not found, but over 60 of age group was decreased in fat parameters than those of forties and fifties. Side effects were noted in 5\% of enrolled patients, ie itching on injection site. All enrolled subjects showed significantly different 
parameters after 26 week treatment of GH as followings:Decreased; $\operatorname{VAT}(-8.6 \%, \mathrm{p}=0.004)$, SAT( $(-6.1 \%, \mathrm{p}=0.050)$, Total abdominal fat $(-7.4 \%, p=0.011)$, AGHDA score $(-1.6, p=0.022$, Increased;HbA1c $(0.2 \%, \mathrm{p}=0.001)$, serum IGF-I $(56 \mathrm{ng} / \mathrm{mL}, \mathrm{p}=0.002)$, Not changed; Fat mass $(-0.6 \mathrm{~kg}), \mathrm{LBM}(+0.3 \mathrm{~kg})$, Abdominal circumference $(-4.3$ $\mathrm{cm})$, Total cholesterol $(-5 \mathrm{mg} / \mathrm{dL})$, LDL-cholesterol $(-1 \mathrm{mg} / \mathrm{dL})$, HDL-cholesterol $(0 \mathrm{mg} / \mathrm{dL})$, Triglyceride $(-2 \mathrm{mg} / \mathrm{dL})$, FBS $(-10$ $\mathrm{mg} / \mathrm{dL}$ ). GH replacement therapy was effective to central obesity patients on visceral, subcutaneous fat reduction, improved QoL by AGHDA score reduction, IGF-I, and HbAlc elevation, but HbAlc was only $0.2 \%$ elevated from $5.4 \%$.

\section{5-4}

\section{Jugular venous ACTH estimation for diagnosis of ACTH dependent Cushing syndrome: Ultrasound guided direct jugular vein sample collection}

Jaya P. Sahoo (1), Ariachery C. Ammini (1), Ashu S. Bhalla (1), Nandita Gupta (1)

(1)All India Institute of Medical Science, New Delhi, India

Aim of study: To assess the utility of internal jugular vein (ijv)/ peripheral ACTH gradient in determining the etiology of ACTH dependent Cushing syndrome. Subjects and Methods: Patients with ACTH dependent Cushing syndrome (except children less than 12 years) had ijv blood collection under ultrasound guidance using a linear $7 \mathrm{MHZ}$ probe. Blood was collected with a $21 \mathrm{G}$ needle at the level of mandible with the patient in supine position. Six ml of blood was collected sequentially from right and left internal jugular veins for ACTH and prolactin estimation. Peripheral blood for ACTH and prolactin was taken from a previously placed IV cannula in the antecubital vein. Results: Thirty patients (20F, 10M, age: 14 to 50) were enrolled for this study. Source of ACTH excess was pituitary in 19, ectopic ACTH in 4 and unknown in 7 cases. Thirteen out of nineteen Cushing disease patients were correctly identified with IJVS. However, one out of four ectopic Cushing had IJVS C: P ratio > 1.6. Overall, it had sensitivity of $68 \%$ with specificity of $75 \%$. In contrast, MRI pituitary and HDDST had sensitivity of $90 \%$ and $58 \%$ respectively, with specificity of $100 \%$ each. Conclusion: Ultrasound guided IJV sample collection may be a useful test for diferential diagnosis of patients with ACTH dependent Cushing syndrome specially in resource poor countries. Simultaneous IJV and peripheral sample collection with CRH stimulation may improve sensitivity and specificity of this test

\section{CVEM 1}

March 27 (Sat.) 9:20-10:10

Room 17

\section{6-1}

Impaired recovery of blood flow after hind-limb ischemia in mice lacking guanylyl cyclase-A, a receptor for atrial and brain natriuretic peptides

Takeshi Tokudome (1), Ichiro Kishimoto (2), Kazuwa Nakao (3), Kenji Kangawa (2)

(1)Division of Hypertension, National Cardiovascular Center Research Institute, Japan, (2)National Cardiovascular Center Research Institute, (3)Department of Medicine and Clinical Science, Kyoto University Graduate School of Medicine, Japan

OBJECTIVE: Atrial and brain natriuretic peptides (ANP and BNP, respectively) function via guanylyl cyclase (GC)-A, resulting in diuresis, natriuresis, and blood vessel dilation. Here, we investigated the role of endogenous ANP/BNP-GC-A signaling on reparative vascular remodeling using a hind-limb ischemia model. METHODS AND RESULTS: In GC-A-deficient mice (GC-A-KO), hind-limb ischemia resulted in autoamputation or severe ulcers in $60 \%$ of mice (6/10) during the 28-day observation period. In wild-type (WT) mice, partial amputation or mild ulcers were detected in only $20 \%$ of mice (2/10). Laser Doppler perfusion imaging revealed that the recovery of blood flow in the ischemic limb was significantly inhibited in GC-A-KO mice compared with WT mice. Immunostainings with anti-PECAM-1 antibody demonstrated that, in GC-A-KO, the capillary density of the ischemic tissue was significantly diminished compared to WT. Furthermore, bone marrow transplantation showed the predominant role of GC-A on local ischemic tissue rather than on vascular progenitor cells mobilized from bone marrow during vascular remodeling. In cultured human endothelial cells, ANP treatment significantly stimulated mRNA expressions of vascular endothelial growth factor and endothelial nitric oxide synthase via Erk1/2dependent mechanism. CONCLUSIONS: These results suggest that endogenous ANP and BNP play important roles in reparative vascular remodeling in ischemic tissue.

\section{6-2}

Atrial and brain natriuretic peptides-guanylyl cyclase-A signaling pathway inhibits TRPC6-mediated pro-hypertrophic signaling in the hearts

Hideyuki Kinoshita (1), Koichiro Kuwahara (1), Motohiro Nishida (2), Zhong Jiang (3), Xianglu Rong (1), Shigeki Kiyonaka (4), Yoshihiro Kuwabara (1), Hitoshi Kurose (2), Ryuji Inoue (3), Yasuo Mori (4), Yuhao Li (1), Yasuaki Nakagawa (1), Satoru Usami (1), Kenji Ueshima (5), Kazuwa Nakao (1)

(1)Department of Medicine and Clinical Science, Kyoto University Graduate School of Medicine, Kyoto, Japan, (2)Department of Pharmacology and Toxicology, Graduate School of Pharmaceutical Sciences, Kyusyu University, Fukuoka, Japan, (3)Department of Physiology, Graduate School of Medical Sciences, Fukuoka University, Fukuoka, Japan, (4)Department of Synthetic Chemistry and Biological Chemistry, Kyoto University Graduated School of Engineering, Kyoto, Japan, (5)Department of EBM Research Center, Kyoto University Graduate School of Medicine, Kyoto, Japan

Rationale; Atrial and brain natriuretic peptides (ANP and BNP, respectively) exert anti-cardiac hypertrophy effects via their common receptor, guanylyl cyclase-A (GC-A), which catalyzes the synthesis of cGMP, leading to activation of protein kinase $\mathrm{G}$ (PKG). Still, much of the network of molecular mediators via which ANP/ BNP-GC-A signaling inhibit cardiac hypertrophy remains to be characterized.

Objective: We investigated the effect of ANP-GC-A signaling on transient receptor potential subfamily C (TRPC) 6, a receptor-operated $\mathrm{Ca}^{2+}$ channel known to positively regulate prohypertrophic calcineurin-nuclear factor of activated T cells (NFAT) signaling.

Methods and Results: In cardiac myocytes ANP phosphorylated TRPC6 at threonine 69 , the PKG phosphorylation site, and significantly inhibited agonist-evoked NFAT activation and $\mathrm{Ca}^{2+}$ influx, while in HEK293 cells, it dramatically inhibited agonist-evoked TRPC6 channel activity. These inhibitory effects of ANP were abolished in the presence of specific PKG inhibitors or by substituting an alanine for threonine 69 of TRPC6. In model mice lacking GC-A the calcineurin-NFAT pathway is constitutively activated, and BTP2, a selective TRPC channel blocker, significantly attenuated the cardiac hypertrophy otherwise seen. Conversely, overexpression of TRPC6 in mice lacking GC-A exacerbated cardiac hypertrophy. BTP2 also significantly inhibited angiotensin II-induced cardiac hypertrophy in mice.

Conclusions: Collectively, these findings suggest that TRPC6 is a critical target of antihypertrophic effects elicited via the cardiac ANP/BNP-GC-A pathway, and suggest TRPC6 blockade could be an effective therapeutic target for preventing pathological cardiac remodeling.

\section{6-3}

Serum insulin-like growth factor-I and its binding protein 3 in their relation to intima media thickness: Results of the Study of Health in Pomerania (SHIP)

Nele Friedrich (1), Elisabeth Spilcke-Liss (1), Marcus Doerr (2), Ulf Schminke (3), Henry Voelzke (4), Georg Brabant (5), Matthias Nauck (1), Henri Wallaschofski (1)

(1)Institute of Clinical Chemistry and Laboratory Medicine, University of Greifswald, Germany, (2) Department of Cardiology, University of Greifswald, Germany, (3)Department of Neurology, University of Greifswald, Germany, (4)Institute for Community Medicine, University of Greifswald, Germany, (5) Department of Endocrinology, Christie Hospital, Manchester, UK

Objective The aim of the present study was to assess the association of the insulin-like growth factor 1 (IGF-I) and its binding protein 3 (IGFBP-3) with the carotid intima-media thickness (IMT) in a population based cohort older than 45 years.Methods In the Study of Health in Pomerania (SHIP), a cross-sectional survey, a total of 2,286 participants $(1,122$ women) aged 45 year or older with readable ultrasound of the carotid arteries were available for the present analyses. Serum IGF-I and IGFBP-3 levels were categorized 
into three groups (low, moderate, high) according to the sex-specific 10th and 90th percentile. Analyses of variance (ANOVA) and logistic regression analyses adjusted for age, waist circumference, diabetes, hypertension, creatinine clearance, and noticeable lipids were performed.Results In men, multivariable ANOVA decreasing IGFBP-3 levels and rising IGF-I/IGFBP-3 ratio were related to increasing IMT. Logistic regression analyses also showed that low IGFBP-3 levels were associated to higher odds of increased IMT, whereas a low IGF-I/IGFBP-3 ratio was accompanied by lower odds of increased IMT. Among women no statistical significant relation between IGF-I, IGFBP-3 as well as IGF-I/IGFBP-3 ratio and IMT as continuous were found. However, high IGF-I were related to an increased IMT. Conclusion In summary, associations between high IGF-I or low IGFBP-3 and IMT were revealed in a populationbased study.

\section{6-4}

\section{RANKL System contributes the process of vascular} calcification as well as osteoporosis

\section{Mariana Osako (1), Hironori Nakagami (2), Ryuichi Morishita (1)}

(1)Department of Clinical Gene Therapy, Osaka University, Osaka, Japan, (2)Division of Gene Therapy Science, Osaka University, Osaka, Japan

RANKL, its receptor RANK, and decoy receptor OPG are key molecules in bone metabolism, which have been recently reported to be present in vasculature, likely working in vascular calcification, since OPG-/- mice show osteoporosis and vascular calcification, similar to postmenopausal women. In this study, we focused on the RANKL signaling as the common molecular mechanism between both diseases, and found that RANKL may contribute to calcification of human aortic smooth muscle cell (HASMC) through induction of the calcification inducer bone morphogenetic protein-2 (BMP-2) by human aortic endothelial cell (HAEC). RANKL also decreased the expression of the calcification inhibitor matrix Gla protein (MGP) in HASMC that may support the function of BMP-2, leading to bonerelated gene expression and calcium deposition increase followed by the osteogenic differentiation of HASMC. We further developed a novel model of vascular calcification with osteoporosis induced by estrogen deficiency (ovariectomy) and western diet for 3 months in ApoE-deficient mice, which demonstrated increased expression of RANKL, RANK and osteopontin by Real Time PCR and in situ hybridization of aorta samples. Western diet induced atherosclerosis, but only ovariectomized mice showed calcification, which was accompanied by RANK, RANKL, BMP-2 and alkaline phosphatase increase, and MGP decrease in vasculature. Importantly, estrogen inhibited RANKL signaling in vitro and the replacement inhibited vascular calcification and osteoporosis in vivo. This study demonstrates the contribution of RANKL signaling to aortic calcification as well as osteoporosis, and indicates that the beneficial effect of estrogen replacement in vascular calcification may be explained by inhibition of RANKL signaling.

\section{CVEM 2}

0 7-1

\section{Involvement of bone morphogenetic protein system in endothelin- and aldosterone-induced cell proliferation of pulmonary arterial smooth muscle cells isolated from pulmonary arterial hypertension}

Fumio Otsuka (1), Ryutaro Yamanaka (1), Kazufumi Nakamura (2), Misuzu Yamashita (1), Hiroyuki Otani (1), Masaya Takeda (1), Jiro Suzuki (1), Tomoko Miyoshi (1), Kenichi Inagaki (1), Toshio Ogura (1), Hirofumi Makino (1) (1)Department of Medicine and Clinical Science, Okayama University Graduate School of Medicine, Dentistry and Pharmaceutical Sciences, Japan, (2)Department of Cardiovascular Medicine, Okayama University Graduate School of Medicine, Dentistry and Pharmaceutical Sciences, Okayama, Japan

The pathology of pulmonary arterial hypertension $(\mathrm{PAH})$ is characterized by the remodeling of pulmonary arteries due to pulmonary artery smooth muscle cell (PASMC) hyperproliferation; however, the detailed mechanism has yet to be elucidated. We investigated the functional link of BMP system and other vasoactive factors associated with PAH in the mitotic actions of PASMCs isolated from idiopathic/secondary PAH lungs. ET1 and aldosterone stimulated PASMC proliferation of idiopathic PAH more efficaciously than that of secondary PAH, whereas Ang II and ET3 failed to activate mitosis in either cell type of PASMC. The effects of ET1 and aldosterone were blocked by an ETA/BR antagonist bosentan and a selective MR blocker eplerenone, respectively. BMP-2 and -7 but not BMP-4 and -6 significantly increased cell mitosis in both cell types of PASMCs. ET1- and aldosterone-induced mitosis as well as MAPK phosphorylation were significantly increased in the presence of BMP-2 and -7 in PASMC isolated from idiopathic PAH, although the additive effects were not observed in PASMC isolated from secondary PAH. Inhibition of ERK1/ERK2 signaling suppressed basal, ET1- and aldosterone-induced PASMC mitosis more potently than that of SAPK/JNK inhibition. Given that BMP-2 and -7 upregulated ETA/BR and MR and that BMP-2 decreased 11betaHSD2 in PASMC isolated from idiopathic PAH, BMPR-Smad signaling may play a key role in amplifying the ETA/BR- and/or MR-ERK signaling in PASMCs of PAH lung. Collectively, the functional link between BMP and ET and/or MR system is involved in the progress of PASMC mitosis, leading to the development of clinical PAH.

\section{$07-2$}

\section{Epigenetic transcriptional repression of the human CYP11B2 gene}

Masashi Demura (1), Fen Wang (1), Takashi Yoneda (1), Shigehiro Karashima (1), Yuan Cheng (1), Masakazu Yamagishi (1), Yoshiyu Takeda (1)

(1)Kanazawa University, Kanazawa, Japan

Aldosterone plays a pivotal role in the development of cardiovascular tissue fibrosis. Aldosterone synthase encoded by CYP11B2 catalyzes final two steps from corticosterone to aldosterone. The Ad1(-71/-64) and Ad5(-129/-114) cis-acting elements are required for the transcriptional regulation of CYP11B2. These elements contain $\mathrm{CpG}$ dinucleotides, which are target sites for DNA methylation. Methylation ratios of Ad1 and Ad5 in aldosterone-producing adenoma $(n=9)$ were about half those of adrenal tumor $(n=5)$, normal $\operatorname{adrenal}(n=4)$ or $\operatorname{artery}(n=3)$. Inverse significant nonparametric correlations were found between methylation ratios and the CYP11B2 mRNA levels. Luciferase-reporter assays showed that CpG methylation completely abolished CYP11B2 promoter activity. Promoter constructs with incomplete methylation weakly responded to stimulation by angiotensin II, KCL or cAMP, suggesting that CYP11B2 promoter activity depended upon methylation. NoShift transcriptional factor assays demonstrated that $\mathrm{CpG}$ methylation significantly decreased CREB binding to the Ad1 by $90 \%$ and NURR 1 binding to the Ad5 by half in nuclear extracts from adrenocortical H295R cells. Likewise, $\mathrm{CpG}$ methylation significantly increased methyl-CpG binding protein 2 (MECP2) binding to the Ad1 in vitro. Chromatinimmunoprecipitation-quantitative PCR showed that MECP2 interacted strongly with methylated Ad1 and weakly with methylated Ad5 in vivo. Other methyl-CpG binding repressors including MBD1 and 2 appeared to bind to neither Ad1 nor Ad5. Taken together, CpG methylation repressed human CYP11B2 promoter activity by decreasing binding of activators as CREB and NURR1 and increasing binding of a repressor as MECP2.

0 7-3

An alternative pathway of mineralocorticoid receptor transactivation by macrophage secretory products

Takeshi Hayashi (1), Hirotaka Shibata (1), Isao Kurihara (1), Yuko Mitsuishi (1), Ayano Murai-Takeda (1), Rie Jo (1), Yuichiro Motosugi (1), Hiroshi Itoh (1) (1)Department of Internal Medicine, School of Medicine, Keio University, Tokyo, Japan

[Aim] Elevation of plasma aldosterone level or mineralocorticoid receptor (MR) activation is involved in resistant hypertension, vascular inflammation and cardiac remodeling. The recent report (Hypertension, 2009) showed that the macrophage MR plays a crucial role in blood pressure elevation and cardiac fibrosis using macrophage-specific MR knockout mice. However, it is unclear how 
infiltrated macrophages induce inflammation and fibrosis in other cell types. We therefore tested the hypothesis that macrophage secretory products directly stimulate MR transcriptional activity.[Methods] We used a human monocytic leukemia cell line, THP-1, which differentiates into macrophages when treated with phorbol 12-myristate 13-acetate. We utilized coculture of THP-1 and COS-7 cells in which MR and MR-responsive reporter plasmids were transfected. We also investigated the effect of transfer of the monocyte-macrophage conditioned media from single culture of THP-1 cells into COS-7 cells on the MR transcriptional activity. [Results] Transient transfection in COS-7 cells showed that $10^{-10} \mathrm{M}$ aldosterone increased MR transactivation by 12 -fold. The MR transcriptional activity was minimally affected with coculture of THP-1-monocytes, whereas significantly enhanced by 4 -fold with coculture of THP-1-macrophages. The THP-1 conditioned media transfer experiments confirmed that macrophage conditioned media enhanced MR tranactivation by 5.2fold, whereas repressed glucocorticoid receptor and androgen receptor transactivation by 0.7 - and 0.4 -fold, respectively, indicating that enhancing effects of macrophage-conditioned media on MR activity is selective. [Conclusion] Macrophage secretory products selectively enhance aldosterone-induced MR transactivation that may contribute to cardiovascular inflammation and fibrosis by infiltrated macrophages. This alternative pathway may account for aldosterone-independent MR activation with normal plasma aldosterone level.

\section{7-4}

\section{Role of mineralocorticoid receptor/Rho/Rho-kinase pathway in obesity-related renal injury}

Hirobumi Tokuyama (1), Shu Wakino (1), Yoshikazu Hara (1), Naoki Washida (1), Satoru Tatematsu (1), Kyoko Yoshioka (1), Kazuhiro Hasegawa (1), Hitoshi Minakuchi (1), Koichiro Homma (1), Koichi Hayashi (1), Hiroshi Itoh (1) (1)Internal Medicine, Keio University, Tokyo, Japan

Both aldosterone and Rho-kinase are demonstrated independently to play an important role in renal injury and impaired insulin signalling. We therefore examined whether aldosterone/Rho/Rho-kinase pathway contributed to obesity-associated nephropathy. C57BL/6J mice were fed a high fat (HFD) or low fat diet (LFD) and mice on HFD were treated with a mineralocorticoid receptor (MR) antagonist, eplerenone. The mice on HFD developed obesity, and hyperglycemia compared with those on LFD, but manifested similar levels of systolic blood pressure. In HFD-fed mice, glomerular hypercellularity and increased mesangial matrix were noted, which paralleled the increase in albuminuria (HFD, $0.13 \pm 0.04 \mathrm{mg} / \mathrm{gCr}, \mathrm{P}<0.01$ vs LFD, $0.02 \pm 0.01 \mathrm{mg} / \mathrm{gCr})$. The treatment with eplerenone alleviated the histological changes and albuminuria $(0.04 \pm 0.01 \mathrm{mg} / \mathrm{gCr}, \mathrm{P}<0.01$ vs HFD) without alterations in systemic blood pressure. Adipose tissue weight and adipocyte size of HFD-fed mice were increased, which were ameliorated by eplerenone. Furthermore, enhanced Rho-kinase activity was noted in kidneys $(2.0$-fold, $\mathrm{p}<0.05)$ and adipose tissues (1.8-fold, $\mathrm{p}<0.05)$ from HFD-fed mice, as well as increased expressions of MCP-1, TNF- $\alpha$ and PDGF-B. All of these changes were attenuated by eplerenone. In HFD-fed mice, MR protein levels in the nuclear fraction were increased in kidneys $(2.3$-fold, $\mathrm{p}<0.05)$ and adipose tissues $(2.0$-fold, $\mathrm{p}<0.05)$ without elevation in serum aldosterone levels (LFD, 278 $\pm 14 \mathrm{mg} / \mathrm{dl}, \mathrm{HFD}, 297 \pm 12 \mathrm{mg} / \mathrm{dl}$ ). Finally, in mesangial cells, stimulation with aldosterone increased Rho-kinase activity, and pre-incubation with eplerenone prevented the aldosterone-induced activation of Rho-kinase. In conclusion, excess fat intake causes obesity and renal injury in $\mathrm{C} 57 \mathrm{BL} / 6 \mathrm{~J}$ mice, and these changes are mediated by enhanced MR/Rho/Rho-kinase pathway and subsequent inflammatory process.

\section{Adrenal 1}

March 27 (Sat.) 11:10-12:00

Room 17

0 8-1

\section{The CYP17A1 gene polymorphisms are associated with} hypertension and bone mineral density

Masayoshi Soma (1), Kaoru Sugama (1), Kotoko Kosuge (1), Takahiro Ueno (2), Noriko Aoi (4), Tomohiro Nakayama (5), Nobutaka Doba (3), Shigeaki Hinohara (3) (1)The Division of General Medicine, The Department of Internal Medicine, Nihon University School of Medicine, Tokyo, Japan, (2)The Division of Nephrology, Hypertension and Endocrinology, The Department of Internal Medicine, Nihon University School of Medicine, Japan, (3)Life Planning Center, Tokyo, Japan, (4)Division of Genomic Epidemiology \& Clinical Trials, The Department of Advanced Medicine, Nihon University School of Medicine, Japan, (5)Division of Laboratory Medicine, Department of Pathology \& Microbiology, Nihon University School of Medicine, Japan

The CYP17A1 gene encodes steroid 17- $\alpha$-hydroxylase, which mediates both 17- $\alpha$-hydroxylase and 17, 20-lyase activity. These functions allow the adrenal glands and gonads to synthesize both $17-\alpha-$ hydroxylated glucocorticoids and sex steroids. The deficiency of this enzyme is characterized by hypertension and impaired production of sex hormones. We performed the studies of the CYP17A1 gene to examine the association with hypertension, serum steroids levels and bone mineral density (BMD) using four tagSNPs (rs743572, rs 1004467, rs17115100 and rs2486758) of the gene in two independent cohorts. Two hundred seventy seven patients with EH and 280 subjects with normal blood pressure (NT) were enrolled to examine the association with hypertension, and 316 subjects with healthy elder Japanese were enrolled to measure serum steroids levels and BMD. Genomic DNA was extracted from peripheral blood leukocytes. Genotypings of 4 SNPs in the gene were done with a TaqMan method. The genotype and allele frequencies of rs 743572 in the CYP17A1 gene is significantly different between the NT and EH groups $(p=0.0036$ and $p=0.0006$, respectively). Serum free testosterone levels are significantly different in women with each genotype of rs 743572 , rs 1004467 or rs17115100. The genotype of rs17115100 significantly influences BMD in women. The genotypes of rs10044467 and rs2486758 significantly affects DHEA-S levels in men. The gene polymorphism of the CYP17A1 may influence to the development of hypertension, and to the serum steroids levels and BMD in elder Japanese.

\section{8-2}

\section{Common genetic variations in the glucocorticoid pathway}

Tomoatsu Mune (1), Tetsuya Suwa (2), Jun Takeda (2), Michihiro Matsuki (1), Kohei Kaku (1)

(1)Division of Endocrinology and Diabetes, Department of Internal Medicine, Kawasaki Medical School, Japan, (2)Department of Diabetes and Endocrinology, Gifu University, Japan

Cortisol is an unique glucocorticoid (GC) essential for maintaining human life. Variations in the GC action have been implicated in the etiology of common diseases such as hypertension, diabetes, obesity, etc. Here we investigated common polymorphisms in a series of genes on the GC pathway in 1442 subjects including 486 hypertensives and 368 type 2 diabetes, and analyzed their associations with diseases and clinical measures. Target genes were CYP17, CYP11B1 (the final step of cortisol production), HSD11B1 (cortisol regenerating enzyme) and $H 6 P D$ (NADPH supplying system) as a prereceptor enhancer of GC action, and $\mathrm{NR} 3 \mathrm{Cl}$ coding the GC receptor. The cc genotype of CYP17 His46c/t was frequent in hypertensives $(\mathrm{P}=$ $.025)$, and the tt genotype of HSD11B1 Int4_1345t/g was frequent in type 2 diabetes $(\mathrm{P}=.021)$. Plasma cortisol was higher in the cc genotype of HSD11B1 Int4_1705c/t. Plasma ACTH was higher in the QQ genotype of $H 6 P D \mathrm{R} 453 \mathrm{Q}$. Urinary cortisol excretion was higher in the QQ genotype of CYP11B1 R43Q. Plasma insulin was lower in the QQ genotype of CYP11B1 R43Q and HbA1c was higher in the QQ genotype of H6PD R453Q. Total cholesterol was higher in the aa genotype of H6PD Ala212g/a, and HDL cholesterol was higher in the gg genotype of HSD11B1 Int4_1345t/g. Visceral versus subcutaneous fat volume was higher in the RR genotype of CYP11B1 $\mathrm{R} 43 \mathrm{Q}$ and the $\mathrm{t} / \mathrm{t}$ genotype of HSD11B1 Int4_1345 t/g. Thus, com- 
mon genetic variants in this pathway are associated with blood pressure homeostasis, glucose tolerance and lipid metabolism.

0 8-3

Discrimination P450 oxidoreductase deficiency from classical 21-hydroxylase deficiency in Japanese infants using urinary $11 \beta$-hydroxyandrosterone by gas chromatography / mass spectrometry

Yuhei Koyama (1,2), Keiko Homma (3), Maki Fukami (4), Reiko Horikawa (5), Masayuki Miwa (6), Kazushige Ikeda (6), Mitsuru Murata (1), Tsutomu Ogata (4), Tomonobu Hasegawa (6)

(1)Department of Laboratory Medicine, Keio University School of Medicine, Tokyo, Japan, (2)Mitsubishi Chemical Medience Co, Tokyo, Japan, (3)Central Clinical Laboratories, Keio University Hospital, Tokyo, Japan, (4) Department of Endocrinology and Metabolism, National Research Institute for Child Health and Development, Tokyo, Japan, (5)Division of Endocrinology and Metabolism, National Center for Child Health and Development, Tokyo, Japan, (6)Department of Pediatrics, Keio University School of Medicine, Tokyo, Japan

Introduction: We have reported that $\mathrm{P} 450$ oxidreductase deficiency (PORD) could be diagnosed biochemically by gas chromatography/ mass spectrometry (GC/MS) as follows; First, differentiation of PORD and/or classical 21-hydroxylase deficiency (C21OHD) from control and/or transient hyper 17OHPnemia using urinary pregnanetriolone (Ptl). Second, discrimination of PORD from $\mathrm{C} 21 \mathrm{OHD}$ using the ratio of urinary $11 \beta$-hydroxyandrosterone (11HA) and pregnanediol (PD). However, in newborn, the measurement of PD was sometimes problematic due to interference by unknown steroids from fetal cortex.

Objective: To explore novel biochemical index to discriminate PORD from C21OHD.

Subjects: We recruited Japanese infants with PORD: 11 cases (newborn: 4, 31-200 days of age: 7), and with C21OHD: 54 cases (newborn: 48, 31-200 days of age: 6). All cases of PORD and C21OHD had been differentiated from control and transient hyper 17OHPnemia using Ptl.

Methods: Sixty three urinary steroid metabolites ( $\mathrm{mg} / \mathrm{g}$ creatinine) were analyzed by GC/MS as previously described with improved specificity

Results: The measurement of PD was interfered in 36/52 of newborn infants. In contrast, the measurement of 11HA, metabolite of $11 \beta$-hydroxyandrostendione, was not interfered in newborn infants at all. 11HA concentration was 0.022-0.217 (PORD) and 0.60923.728 (C21OHD), respectively. PORD and C21OHD were discriminated completely by using tentative $11 \mathrm{HA}$ cutoff point; 0.35 .

Discussion: Urinary 11HA measurement was not interfered by unknown steroids from fetal cortex. Using urinary 11HA, we could completely discriminate PORD from $\mathrm{C} 21 \mathrm{OHD}$ in infants including newborn.

0 8-4

Mutational analysis of NF-1 gene in a family of neurofibromatosis type 1 and pheochromocytoma

Hyon Seung Yi (1), Jung Mi Lim (1), Le Byung Park (1), Yeun Sun Kim (1), Suntaek Hong (2), Sihoon Lee (1)

(1)Department of Internal Medicine, Laboratory of Molecular Endocrinology, Gachon University of Medicine and Science, Incheon, Republic of Korea, (2)Laboratory of Cancer Cell Biology, Lee Gil Ya Cancer and Diabetes Institute, Gachon University of Medicine and Science, Incheon, Republic of Korea

Neurofibromatosis type 1 (NF1) is one of the most common autosomal dominant inherited disorder affecting nervous system and is associated with mutations in the NF1 gene, which is located on chromosome sub-band 17q11.2 and contains 57 exons spanning approximately $300 \mathrm{~kb}$ of genomic DNA. NF1 is caused by a loss of function mutation of the NF1 gene resulting in inactivation of neurofibromin, as a tumor suppressor, which encodes a GTPase activating protein (GAP) involving in the negative regulation of Ras activity. GAP related domain, which is encoded by exons $20-27$ a, and cysteine-serine rich domain are well known to be mostly important functional domains in neurofibromin. In spite of many genetic analysis of NF1 related pheochromocytoma, there still remains the question of whether pheochromocytoma is a true component of NF1. We found a novel germline mutation of NF-1 gene $(\mathrm{c} .7907+1 \mathrm{G}>\mathrm{A})$ in a 31-year-old female patient with NF-1 accompanied by pheochromocytoma and genetic analyses of her family members showed that this mutation leads to skipping of exon 45 during NF1 mRNA splicing. As this novel germline mutation falls into neither GAP related domain nor cysteine-serine rich domain but into $\mathrm{C}$-terminal region of NF1 gene, which is relatively not well known for its function, this suggests that there must be essential function in the C-terminal region of NF1 gene, especially in the development of NF-1 related pheochromocytoma. Therefore, we should necessarily consider the mutation of C-terminal region in NF1 gene when we encounter the patient with NF1 accompanied by pheochromocytoma.

\section{Diabetes mellitus 3}

March 28 (Sun.) 11:10-12:00

Room 11

09-1

Prevalence of chronic complications of type 2 diabetes mellitus patients: Based on the Korean National Diabetes Program

Sang Youl Rhee (1,2), Min Chul Choi (3), Suk Chon (2,4), Sei Hyun Baik (5), Kwan Woo Lee (6), Moon Suk Nam (7), Yong Soo Park (8), Jeong-taek Woo (2,4), Young Seol Kim $(2,4)$

(1)Department of Endocrinology and Metabolism, The Armed Forces Capital Hospital, Gyeonggi, Republic of Korea, (2)Research Institute of Endocrinology, Kyung Hee University, Republic of Korea, (3)Department of Occupational and Environmental Health, School of Public Health, Seoul National University, Republic of Korea, (4)Department of Endocrinology and Metabolism, Kyung Hee University School of Medicine, Republic of Korea, (5)Division of Endocrinology and Metabolism, Department of Internal Medicine, Korea University, Republic of Korea, (6)Department of Endocrinology and Metabolism, Ajou University School of Medicine, Gyeonggi, Republic of Korea, (7)Department of Internal Medicine, Inha University School of Medicine, Gyeonggi, Republic of Korea, (8)Department of Internal Medicine, Hanyang University College of Medicine, Republic of Korea

Objective: Korean National Diabetes Program (KNDP) is a nationwide, large-scale, prospective, multicenter cohort study to investigate the pathophysiology of Korean type 2 diabetes mellitus (T2DM) patients. In this sub-study, we evaluated the prevalence of diabetes related chronic complications in Korean T2DM subjects based on KNDP registered subjects.Research Design and Methods: We enrolled total 4,265 KNDP registered subjects in 13 major university hospitals in Korea. Among them, we analyzed the crude prevalence of macrovascular and microvascular complications in 3,795 subjects and calculate standardized prevalence according to the Korean National Health and Nutrition Survey (KNHANES) 2005 subjects. Results: Among KNDP subjects, crude prevalence of hypertension was $43.2 \%$ and Dyslipidemia was $34.8 \% .10 .8 \%$ of subjects had macrovascular complications and $16.7 \%$ had microvascular complications. When adjusted by KNHANES 2005 population, standardized prevalence of chronic complications in KNDP subjects was decreased. However, standardized prevalence ratios (SPR) of KNDP subjects were significantly higher than KNHANES 2005 subjects except for the SPR of myocardial infarction and angina pectoris in women. Conclusion: Prevalence of macrovascular and microvascular complications in Korean T2DM patients was significantly higher than normal population. However, prevalence of cardiovascular complications in women is not significantly different.

0 9-2

Obesity and metabolic abnormalities in children and grand children of subjects with type $\mathbf{2}$ diabetes mellitus

Ariachery C Ammini (1), EDAVAN P PRAVEEN (1), Bindu Kulshresta (1),

Sadanand Dwivedi (1), Madan L Khurana (1), Nandita Gupta (1),

Jayaprakash Sahoo (1)

(1)All India Institute of Medical Sciences, New Delhi, India

Aim: To study the prevalence of obesity, dyslipidemia and glucose intolerance in children and grandchildren of subjects with type 2 diabetes mellitus.Subjects and methods: Children (age 5-20) who had parent or grandparent with type 2 diabetes were studied. All subjects underwent medical history, physical examination, lipid profile and OGTT. Samples were collected for glucose, insulin, C-peptide and Proinsulin estimation.Results: One hundred seventy five of 265 offspring from 94 families, $(90 \mathrm{~m}, 85 \mathrm{f}$,mean age $14.4 \mathrm{yrs})$ were studied. One hundred twenty seven (72.5\%) subjects were lean, $27(15.4 \%)$ subjects were overweight and 21(12\%) subjects were obese. Five 
subjects (2.9\%) had IFG, $8(4.6 \%)$ had IGT and 3(1.7\%) subjects had DM. Among the 8 IGT subjects $6(75 \%)$ subjects were of normal weight. Youngest DM detected was at 8 years of age and youngest IGT was at 12 years of age. Glucose intolerance was higher when both father and mother had $\operatorname{DM}(26 \%)$ compared to subjects whose father, mother or grandparents had DM(3-7\%). Lipid abnormalities were observed as early as 6 years of age. $16 \%$ of subjects had abnormal Triglycerides, $18 \%$ of subjects had abnormal total cholesterol, $28 \%$ subjects had abnormal HDL-cholesterol. About $50 \%$ of subjects of 5-10 age group and 11-20 age group had at least one lipid abnormality. Conclusion: Overweight, dyslipidemia and glucose intolerance starts during childhood among offspring of subjects with diabetes mellitus. Normal BMI does not prevent glucose intolerance.

\section{9-3}

\section{Diabetic subjects with maternal history of diabetes have a more adverse cardiometabolic profile}

Ronald C. Ma (1), Ying Wang (1), Andrea O. Luk (1), Claudia H. Tam (1), Janice S. Ho (1), Heung M. Lee (1), Maggie C. Ng (1), Wing-yee So (1), Juliana C. Chan (1)

(1)Department of Medicine and Therapeutics, Chinese University of Hong Kong, Hong Kong

Background: Offspring exposed to maternal diabetes or intra-uterine hyperglycaemia have increased risk of diabetes and metabolic syndrome due to fetal programming. In this study, we aimed to compare the impact of maternal and paternal history of diabetes in a large registry of Chinese patients with diabetes. Methods: We reviewed the family history and baseline characteristics of patients from the Hong Kong Diabetes Registry. We included in the analysis 8864 Chinese patients with type 2 diabetes for whom details of parental diabetes status is available. Subjects are classified according to parental history of diabetes into no parental history of diabetes, maternal diabetes, paternal diabetes or bilineal. Baseline characteristics, including age of onset, presence of hypertension, hyperlipidaemia, microalbuminuria and glycaemic control are compared between the different groups. Results: Out of 8864 patients with type 2 diabetes, 5952 have no known parental history of diabetes, whilst 460 subjects have both parents affected with diabetes. Maternal diabetes was reported by 1476 subjects (781 female, 695 male), which is significantly more common than paternal diabetes (776 patients, 393 female, 383 male, $p<0.001$ ). In female offspring, history of maternal diabetes is associated with higher prevalence of hypertension compared with subjects with paternal history of diabetes $(p=0.01)$. Maternal diabetes is associated with high prevalence of hypertension in female compared to male offspring $(\mathrm{p}<0.01)$. Conclusions: Maternal diabetes is associated with a more adverse cardiometabolic risk profile. Offspring exposed to maternal diabetes are at increased risk of diabetes and metabolic syndrome and should be subject to more intensive screening.

\section{9-4}

Integration of 69 publicly-available genome-wide functional experiments discovers a novel gene highly associated with type 2 diabetes

Momoko Horikoshi (1), Kazuo Hara (1), Takashi Kadowaki (1), Keiichi Kodama (2,3), Marina Sirota (2,3), Rong Chen (2,3), Atul J. Butte (2,3,9), Kyoko Toda (4),

Satoru Yamada (5), Junichiro Irie $(5,6)$, Hiroshi Ohtsu $(1,7)$, Shiro Maeda (8)

(1)Department of Diabetes and Metabolic diseases, University of Tokyo, Tokyo, Japan, (2)Stanford Center for Biomedical Informatics Research, Department of Medicine, Stanford University School of Medicine, CA, USA, (3)Department of Pediatrics, Stanford University School of Medicine, CA, USA, (4)Division of Basic Research, Biomedical Laboratory, Kitasato Institute Hospital, Kitasato University, Tokyo, Japan, (5)Diabetes Center, Kitasato Institute Hospital, Kitasato University, Tokyo, Japan, (6)Department of Internal Medicine, Keio University School of Medicine, Tokyo, Japan, (7)Department of Clinical Trial Data Management, Graduate School of Medicine, University of Tokyo, Tokyo, Japan, (8)Laboratory for Endocrinology and Metabolism, Center for Genomic Medicine, RIKEN, Kanagawa, Japan, (9)Lucile Packard Childre's Hospital, CA, USA

Type 2 diabetes (T2D) is a complex, polygenic disease, and phenotypically characterized by insulin resistance. Growing evidence has indicated the causative link between low-grade inflammation in adipose tissue and the development of insulin resistance/T2D. To find causative T2D-associated genes, we meta-analyzed 69 experiments, totaling 518 publicly-available T2D-related microarrays, based on our hypothesis that genes differentially expressed in T2D across models are strong candidates for functional variants. Our top candidate gene, encoding a cell-surface glycoprotein involved in immunological cellcell interactions, was implicated in 46 microarray experiments, and is an unknown gene for T2D susceptibility. We confirmed the strong association of a coding single nucleotide polymorphisms (cSNP) with T2D in 2,830 T2D-cases and 1,928 controls from two independent cohorts $\left(\mathrm{OR}=1.43\right.$ [C.I. 1.17-1.74], $\left.\mathrm{P}=3.9 \times 10^{-4}\right)$. This cSNP shows a cumulative effect with TCF7L2 and KCNQ1 variants, with OR reaching 5.36 with $>=5$ risk alleles $\left(\right.$ Overall $\left.\mathrm{P}=2.1 \times 10^{-7}\right)$. We found that the risk-allele of this cSNP increased its mRNA expression in adipose tissue, and serum levels of this immune-receptor were positively correlated with insulin resistance, both in humans. Importantly, deficiency of this gene in a mouse model suppresses mononuclear cell infiltration into adipose tissue, and ameliorates insulin resistance. Our findings demonstrate a novel gene genetically and functionally implicated in T2D, and integration of multiple microarray experiments may yield functional variants for complex diseases.

\section{Obesity and adiposcience 1}

March 28 (Sun.) 11:10-12:00

Room 12

0 10-1

\section{In vivo imaging reveals adipose tissue remodeling and chronic inflammation in obesity}

Satoshi Nishimura $(1,2,3)$, Mika Nagasaki $(1,4)$, Ichiro Manabe $(1,2,3)$, Koji Eto (5), Ryozo Nagai $(1,2)$

(1)Department of Cardiovascular Medicine, The University of Tokyo, Tokyo, Japan, (2)Translational Systems Biology and Medicine Initiative, The University of Tokyo, Japan, (3)PRESTO, Japan Science and Technology Agency, Japan, (4)Computational Diagnostic Radiology and Preventive Medicine, The University of Tokyo, Japan, (5)Laboratory of Stem Cell Therapy, Center for Experimental Medicine, Institute of Medical Science, The University of Tokyo, Japan

Metabolic syndrome is a major risk factor of cardiovascular events, and obese visceral adipose tissue remodeling and malfunctioning based on chronic inflammation local immunological changes plays a central role. To assess dynamic interplay between multiple cell-types in obese adipose, a visualization technique in vivo was therefore developed to reveal the underlying multi-cellular and molecular mechanisms of obesity. In vivo imaging revealed close spatial and temporal interrelationships between angiogenesis and adipogenesis in obese adipose (2007 Diabetes). In addition, increased leukocyte-platelet-endothelial cell interactions in the microcirculation of obese adipose were observed, a hallmark of inflammation (2008 J Clin Invest). We also found that large numbers of CD8+ effector T cells infiltrated into obese adipose. The infiltration by CD8+ T cells preceded the accumulation of macrophages, and immunological and genetic depletion of CD8+ T cells reduced macrophage infiltration and adipose tissue inflammation, and ameliorated systemic insulin resistance. Conversely, adoptive transfer of CD8+ T cells to CD8-deficient mice aggravated adipose inflammation. Coculture and other in vitro experiments revealed the vicious interactions between CD8+ T cells, macrophages and adipose tissue. Our findings suggest that obese adipose tissue activates CD8+ T cells, which in turn promote the recruitment and activation of macrophages, thereby initiating and maintaining inflammatory cascades in obese adipose tissue. Infiltration by CD8 $+\mathrm{T}$ cells is, thus, essential for the initiation and development of adipose inflammation (2009 Nat Med). Our results clearly demonstrated the power of our imaging technique to analyze complex cellular interplays in vivo and to evaluate new therapeutic interventions against them.

\section{$010-2$}

\section{Post-transcriptional alternative splicing of anti-apoptotic proteins during adipogenesis programs pro-survival pathways in 3T3-L1 adipocytes}

Denise R. Cooper (1), Li Pengfei (1), Eden Kleiman (1), James Watson (2), Tomar Ghansah (1), Niketa A. Patel $(1,2)$

(1)Department of Molecular Medicine, University of South Florida College of Medicine, USA, (2)Research Service, James A. Haley Veterans Hospital, Tampa, FL, USA

3T3-L1 cells provide a model to study adipogenesis in culture. Mature adipocytes resist apoptosis; however, the basis for this is poorly 
understood. Several anti-apoptotic proteins are the result of alternatively splicing. The differentiation of 3T3-L1 pre-adipocytes from days (D)0-10, was studied. Cell lysates, analyzed by SDS-PAGE and immunoblot, showed substantial changes in expression of three alternatively spliced proteins $(\mathrm{PKC} \beta, \mathrm{PKC} \delta$, and $\mathrm{Bcl}-\mathrm{x})$ involved in cellular apoptosis. $\mathrm{PKC} \beta \mathrm{II}$ is pro-survival as it phosphorylates Akt2 on Serine474 [Biochem Biophys Res Commun 388(2009)554-559]. $\mathrm{PKC} \delta$ splice variants are associated with apoptosis and pro-survival

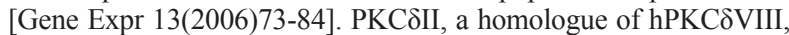
is caspase-3 resistant, and anti-apoptotic; PKC\&deltaI is apoptotic. $\mathrm{Bcl}-\mathrm{x}$ splice variant, $\mathrm{Bcl}-\mathrm{xL}$, is associated with resistance to caspaseinduced apoptosis; Bcl-xS is apoptotic. D0 cells expressed PKC $\beta I$, $\mathrm{PKC} \delta \mathrm{I}$ and $\mathrm{Bcl}-\mathrm{xS}$. D5 cells expressed PKC $\beta \mathrm{II}, \mathrm{PKC} \delta \mathrm{II}$, and Bcl-xL that peaked at $\mathrm{D} 8$ with a concurrent decrease in PKC $\beta \mathrm{I}, \mathrm{PKC} \delta \mathrm{I}$ and Bcl-xS expression. Further, phospho-PTEN, an upstream lipid phosphatase associated with anti-apoptotic cells, was expressed D5-10. The switch in splice variants was accompanied by increased expression of SRp40, SRp55, SC35, and ASF/SF2, SR protein splicing factors. D8-10 cells were protected against serum-starvation induced apoptosis whereas serum-starvation induced DNA laddering on D0, and insulin did not protect cells. In this novel study, the differentiation-induced splicing of anti-apoptotic proteins suggests it is a pivotal switch in adipogenesis that commits cells to differentiation and a pro-survival pathway. By understanding mechanisms of post-transcriptional splicing of anti-apoptotic proteins, it will be possible to program adipogenesis for therapeutic management.

\section{10-3}

\section{DHEA interfere adipogenic differentiation of bone marrow} mesenchymal cells via epigenetic regulation

Krissanapong Manotham (1), Masami Nakatsu (1), Supreecha Chattong (1), Piyapa Kiatsomchai (1), Jaivan Tanamai (1), Pravit Limkuansuwan (1), Anan Sethpakadee (1)

(1)Cell biology and Regenerative Medicine, Department of Medicine, Lerdsin General Hospital, Thailand

Dehydroepiandrosterone (DHEA), a c-19 androgen adrenal hormone, that normally decreases with age,has multiple effects on metabolisms and energy expenditures. Fat accumulation is an age related phenomenon that partially restores by DHEA. Mesenchymal stem cells (MSCs) can differentiate to be adipocyte. However, little is known about the effect of DHEA on adipogenic differentiation of MSCs. In this work, murine mesenchymal stem cells (KUSA O), were undergone adipogenic induction. These cells obtained fat characteristic as well as increased GLUT 4 transcription at day 10 after induction. In a presence with DHEA, fat characteristics were partially inhibited and GLUT4 transcription was repressed. Further study showed no different in proliferation rate and cytotoxicity by DHEA. RT-PCR suggested that DHEA treatment significantly reduced PPAR $\gamma 2$ transcription. In addition, chromatin immunoprecipitation (CHIP)of acetylate Histone 3(H3) suggested that the histone 3 core at the promoter region of PPAR $\gamma$ gene was hypo-acetylated with DHEA. Taken together, these results suggested the novel link between DHEA and the adipogenic differentiation of MSCs.

\section{10-4}

\section{Activating transcription factor 3 constitutes a negative feedback mechanism that attenuates saturated fatty acid/toll- like receptor 4 signaling and macrophage activation in obese adipose tissue}

Takayoshi Suganami (1), Xunmei Yuan (1), Yuri Shimoda (1), Nobutaka Nakagawa (1), Ibuki Shirakawa (1), Yoshihiro Miyamoto (4), Kazuki Yasuda (5), Yasutomi Kamei (1), Shigetaka Kitajima (2), Yoshihiro Ogawa $(1,3)$

(1)Dept. of Molecular Medicine and Metabolism, Medical Research Institute, Tokyo Medical and Dental University, Tokyo, Japan, (2)Department of Biochemical Genetics, Medical Research Institute, Tokyo Medical and Dental University, Tokyo, Japan, (3)Global Center of Excellence Program; International Research Center for Molecular Science in Tooth and Bone Diseases, Tokyo Medical and Dental University, Tokyo, Japan, (4) Department of Medicine, Division of Atherosclerosis and Diabetes, National Cardiovascular Center Hospital, Osaka, Japan, (5)Department of Metabolic Disorder, Research Institute, International Medical Center of Japan, Tokyo, Japan

Obese adipose tissue is markedly infiltrated by macrophages, suggesting that they may participate in the inflammatory pathways that are activated in obese adipose tissue. Evidence has suggested that saturated fatty acids released via adipocyte lipolysis serve as a naturally occurring ligand that stimulates Toll-like receptor 4 (TLR4) signaling, thereby inducing the inflammatory responses in macrophages in obese adipose tissue. Through a combination of cDNA microarray analyses of saturated fatty acid-stimulated macrophages in vitro and obese adipose tissue in vivo, here we identified activating transcription factor 3 (ATF3), a member of the ATF/cAMP response element-binding protein family of basic leucine zipper-type transcription factors, as a target gene of saturated fatty acids/TLR4 signaling in macrophages in obese adipose tissue. Importantly, ATF3, when induced by saturated fatty acids, can transcriptionally repress tumor necrosis factor- $\alpha(\mathrm{TNF} \alpha)$ production in macrophages in vitro. Chromatin immunoprecipitation assay revealed that ATF3 is recruited to the region containing the $\mathrm{AP}-1$ site of the endogenous $\mathrm{TNF} \alpha$ promoter. Furthermore, transgenic overexpression of ATF3 specifically in macrophages results in the marked attenuation of proinflammatory M1 macrophage activation in the adipose tissue from genetically obese $\mathrm{KK} A^{y}$ mice fed high-fat diet. This study provides evidence that ATF3, which is induced in obese adipose tissue, acts as a transcriptional repressor of saturated fatty acids/TLR4 signaling, thereby revealing the negative feedback mechanism that attenuates obesity-induced macrophage activation. Our data also suggest that activation of ATF3 in macrophages offers a novel therapeutic strategy to prevent or treat obesity-induced adipose tissue inflammation.

\section{Thyroid 2}

March 28 (Sun.) 11:10-12:00

Room 13

011-1

Molecular mechanism of negative regulation of the cardiac myosin heavy chain $\beta$ gene by thyroid hormone

Shigekazu Sasaki (1), Hiroyuki Iwaki (1), Akio Matsushita (1), Kenji Ohba (1), Hideyuki Matsunaga (1), Shingo Suzuki (1), Hiroko Misawa (1), Keiko Ishizuka (1), Hirotoshi Nakamura (1)

(1)Hamamatsu University School of Medicine, Japan

In the previous studies of the negative regulation of thyrotropin (TSH) $\beta$ gene by thyroid hormone (T3), we found (1) main transcriptional activator for the TSH $\beta$ gene is GATA2 but not Pit1 (2) although intact DNA binding domain (DBD) of T3 receptor (TR) is important, so-called negative TRE is unnecessary (3) TR-DBD does not bind DNA directly but recognizes GATA2 via proteinsprotein interaction (4) T3-bound TR (T3/TR) interferes with function of GATA2 associated with TRAP220/MED1, a constituent of RNA polymerase II holo-enzyme. Throughout the above analysis, we found that identification of main activator (ex. GATA2 for the TSH $\beta$ gene) is important. In addotion to TSH $\beta$ gene, T3/TR inhibits the gene expression of cardiac myosin heavy chain $\beta$ (MHC $\beta$ ), a molecular marker for heart failure. Two M-CAT elements and an $\mathrm{A} / \mathrm{T}$ rich sequence in the MHC $\beta$ promoter are known to be critical for its expression in the rat cardiac cell primary culture. Using reporter assays with kidney-derived CV1 cells and cardiac musclederived $\mathrm{H} 9 \mathrm{c} 2$ cells and gel shift assays, we demonstrate here that TEF family transcription factors activate the MHC $\beta$ promoter via these elements and that this activities were suppressed by T3/TR. TR was co-immunoprecipitated with TEF-1 via the direct interaction between TR-DBD and TEA domain of TEF-1 in a T3-independent fashion. Over-expression of TRAP220/MED1 enhanced the transactivation by TEF family. Our findings suggested the existence of common mechanisms between the T3-induced transrepressions of the TSH $\beta$ gene and that of the MHC $\beta$ gene. 
$011-2$

\section{Tumor necrosis factor alpha (TNF-a) polymorphisms in Chinese patients with Graves' disease}

Li-Qun Gu (1,2,3), Wei Zhu (1,2,3), Chun-Min Pan (1,2,3), Lin Zhao (1,2,3), Min jia Zhang (1,2,3), Jian-Min Liu (1,2,3), Huai-Dong Song $(1,2,3)$,

Guang Ning (1,2,3), Yong-Ju Zhao $(1,2,3)$

(1)Department of Endocrine and Metabolic Diseases, Rui-jin Hospital, Shanghai Jiao-tong University School of Medicine, China, (2)Shanghai Institute of Endocrine and Metabolic Diseases, China, (3)Shanghai Clinical Center For Endocrine and Metabolic Diseases, China

Objectives: Tumor necrosis factor alpha (TNF- $\alpha$ ) may play a central role in the development of Graves' disease (GD). The aim of this study was to investigate the association of TNF- $\alpha$ polymorphisms with GD in Chinese population.

Design and methods: Genomic DNA was extracted from peripheral blood lymphocyte of 436 GD patients and 316 control subjects. TNF- $\alpha$ polymorphisms at positions -308 (G-308A, rs 1800629), -238 (G-238A, rs361525), and +419 (G+419A, rs3093661) were genotyped.

Results: The distribution of TNF- $\alpha-238$ and +419 allelic frequencies between GD and control individuals was significantly different. Both the $\mathrm{G}$ alleles of TNF- $\alpha-238$ (OR 2.385, 95\%CI 1.359-4.184) and +419 (OR 2.293, 95\%CI 1.303-4.035) SNPs conferred higher risk of GD as compared with A alleles. No significant difference of -308 allelic frequency was observed. Further haplotype analysis revealed that the haplotype GGG was associated with an increased risk of GD (OR 1.554,95\% CI 1.125-2.146), whereas the haplotype GAA was found to be protective(OR 0.419;95\%CI 0.239-0.736). Conclusions: This study demonstrated the association of TNF- $\alpha$ gene with GD in Chinese patients.

\section{1-3}

\section{Changes in serum concentrations of asymmetric dimethylarginine (ADMA), NT-proBNP, leptin and adiponectin in patients with Graves' disease}

Li-Qun Gu (1,2,3), Wei Zhu (1,2,3), Jian-Min Liu (1,2,3), Feng-Ying Li (1,2,3),

Lin Zhao (1,2,3), Min jia Zhang (1,2,3), Yun Liu (1,2,3), Guang Ning (1,2,3),

Yong-Ju Zhao $(1,2,3)$

(1)Department of Endocrine and Metabolic Diseases, Rui-jin Hospital, Shanghai Jiao-tong University School of Medicine, China, (2)Shanghai Institute of Endocrine and Metabolic Diseases, China, (3)Shanghai Clinical Center For Endocrine and Metabolic Diseases, China

Objective: Endothelial dysfunction, as well as abnormal thyroid hormone and adipocytokine levels may be responsible for increased cardiovascular risk in Graves' disease (GD). Asymmetric dimethylarginine (ADMA) and NT-proBNP are new markers of endothelial and myocardiac dysfunction, respectively. The purpose of this study was to investigate the relationship between the serum levels of ADMA with the levels of NT-proBNP, leptin, adiponectin and thyroid hormones in Graves' patients.

Methods and Patients: 239 GD (Female: 182, Male: 57) patients and 81 normal controls were enrolled for main analysis. Another 30 patients with abnormal glucose tolerance were recruited for subgroup analysis. Serum concentrations of thyroid hormones, ADMA, NT-proBNP, leptin and adiponectin were all measured.

Results: Serum levels of ADMA were positively related with FT3 $(\mathrm{r}=0.584, \mathrm{p}<0.001), \mathrm{FT} 4(\mathrm{r}=0.551, \mathrm{p}<0.001)$ and TRAb levels $(\mathrm{r}=0.502$, $\mathrm{p}<0.001)$. Serum NT-proBNP levels were positively associated with FT3 $(r=0.243, p<0.001)$ and FT4 levels $(r=0.274, p<0.001)$, as well as heart rate $(r=0.271, \mathrm{p}<0.03)$. Neither ADMA nor NT-proBNP showed an association with leptin and adiponectin levels. The elevation of serum ADMA and NT-proBNP levels were also observed in patients with controlled hyperthyroidism. The serum ADMA and NT-proBNP levels were not different between GD patients with or without impaired glucose regulation and diabetes.

Conclusion: Serum ADMA and NT-proBNP levels were increased in GD patients independent of adipocytokines. Monitoring these two parameters may be useful in detecting the early changes of endothelial dysfunction, vascular stiffness and fluid volume in GD patients.
$011-4$

\section{A new attempt to establish a quantitative method of real time tissue elastography (RTE) in patients with thyroid and parathyroid disease}

Shinichi Suzuki (1), Keiichi Nakano (1), Toshihiko Fukushima (1),

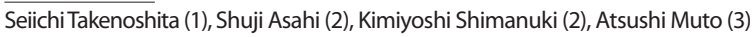
(1)Department of Breast Endocrine Thyroid Surgery, Fukushima Medical University School of Medicine, Fukushima, Japan, (2)Department of Surgery, Aizu Chuo Hospital, Aizu Wakamatsu, Japan, (3)Department of Surgery, Fukushima Rosai Hospital, Iwaki, Japan

We herein report on the utility of using RTE for the diagnosis of both thyroid and parathyroid disease utilizing a new quantitative method, in addition to performing a qualitative evaluation of RTE. The subjects consisted of 207 thyroid and parathyroid patients who were diagnosed based on a qualitative and quantitative evaluation of RTE. Method: All patients underwent elastography accompanied with B-mode ultrasonography using either the EUB8500 or 7500 device (Hitachi Medico.). Elastography images of both thyroid and parathyroid disease were then classified into 4 grades, and these were judged by moving images. In addition, the ratio of a distortion of the sternocleidomastoid muscle as a control and a lesion based on still pictures was quantitatively calculated as the Strain Ratio (SR). Results: Average of SR in FA was 1.0, and averages of SRs in thyroid cancers including PTC, FTC, MTC and ATC were all under 0.4. SRs in thyroid carcinomas were also significantly lower than those in benign thyroid tumors. It is therefore considered to be useful for making a differential diagnosis between thyroid cancer and benign tumors, especially between follicular thyroid cancer and adenoma. Parathyroid adenoma was higher SR than the normal thyroid gland and sternocleid muscle. In conclusion, quantitative method of RTE is therefore considered to be a more useful diagnostic modality for making a differential diagnosis of either thyroid or parathyroid disease.

\section{Pituitary / Neuroendocrinology 2}

March 28 (Sun.) 11:10-12:00

Room 16

$012-1$

\section{Augurin is a novel hypothalamic neuropeptide which} stimulates the hypothalamo-pituitary-adrenal axis

John A. Tadross (1), Michael Patterson (1), Keisuke Suzuki (1),

Mohammad A. Ghatei (1), Stephen R. Bloom (1)

(1)Department of Investigative Medicine, Imperial College London, UK

The functional characterisation of secreted peptides is important as it provides a basis for the development of novel therapeutic agents. Augurin is a recently identified secreted peptide of unknown function. It is expressed in multiple endocrine tissues, and in regions of the brain including the hypothalamus. We investigated the effect of hypothalamic injection of augurin on the hypothalamo-pituitary axes in male Wistar rats. Intracerebroventricular injection of augurin potently increased plasma $\mathrm{ACTH}$ and corticosterone, without a measureable change in other hypothalamo-pituitary axes hormones. Microinjection of lower doses of augurin into the paraventricular nucleus (PVN) caused a similar increase in plasma ACTH and corticosterone, without altering behavioural patterns. Incubation of hypothalamic explants with augurin caused a significant rise in $\mathrm{CRH}$ and arginine vasopressin release, and pre-treatment with a $\mathrm{CRH}$ receptor antagonist prevented the rise in ACTH and corticosterone caused by ICV augurin injection. These data suggest that augurin stimulates the release of ACTH via CRH neurons in the PVN. An antibody raised against synthetic augurin was used to immunohistochemically characterise augurin-like immunoreactivity in the hypothalamus of male Wistar rats. Augurin immunoreactive neurons were found in several regions of the hypothalamus including the PVN, supraoptic nucleus, periventricular nucleus, arcuate nucleus, and the median eminence. This is the first report of a potential physiological role for the neuropeptide augurin in the regulation of the HPA axis. 
$012-2$

Distinct histone-specific modifications mediate positive versus negative transcriptional regulation of TSHa promoter

Paul M. Yen (1), Xianmin Xia (2), Roy Weiss (3), Samuel Refetoff (3), Dongqing Wang (4)

(1)Cardiovascular and Metabolic Diseases Program, Duke-NUS Graduate Medical School, Singapore, (2)Department of Surgery, Tongji Medical College, Wuhan, China, (3)Division of Endocrinology and Metabolism, Dept. of Medicine, University of Chicago, Chicago, IL, USA, (4)Endocrinology Division, Dept. of Medicine, Johns Hopkins University School of Medicine, Baltimore, MD, USA

TSH $\alpha$, a heterodimer partner for thyrotropin (TSH), is secreted by the pituitary gland. T3 negatively regulates $\mathrm{TSH} \alpha$ gene expression via thyroid hormone receptors (TRs) which belong to the nuclear hormone receptor superfamily, whereas TRH positively regulates via the TRH receptor which is a $\mathrm{G}$ protein-coupled receptor. We studied regulation of the TSH $\alpha$ gene by cAMP and T3 using chromatin immunoprecipitation (ChIP) assays in stably-transfected rat pituitary cells containing the human $\mathrm{TSH} \alpha$ promoter. Interestingly, cAMP selectively increased histone $\mathrm{H} 4$ acetylation whereas T3 surprisingly induced histone $\mathrm{H} 3$ acetylation. In particular, cAMP increased H4K5 and H4K8 acetylation and decreased H4K20 trimethylation, modifications associated with transcriptional activation. T3 increased H3K9 and H3K18 acetylation and H3K4 trimethylation; however, it also decreased H3K27 acetylation and increased H3K27 trimethylation which are associated with transcriptional repression. Of note, cAMP recruited $\mathrm{pCREB}, \mathrm{CBP} / \mathrm{p} 300$, and PCAF to the promoter whereas $\mathrm{T} 3$ caused dissociation of $\mathrm{NCoR} / \mathrm{SMRT}$ and HDAC3. Overexpression of a dominant negative mutant thyroid hormone receptor (TR) from a patient with resistance to thyroid hormone (RTH) led to less T3-dependent negative regulation and partially blocked histone $\mathrm{H} 3$ modifications of the $\mathrm{TSH} \alpha$ promoter. Our findings showed that non-overlapping histone-specific modifications determine positive versus negative transcriptional regulation, and integrate opposing hormonal and intracellular signals at the TSH $\alpha$ promoter. A mutant TR from a patient with RTH exerted dominant negative activity by blocking the histone modifications induced by $\mathrm{T} 3$ on the TSH $\alpha$ promoter and likely is a major contributor to the inappropriate TSH production observed in RTH.

$012-3$

\section{Cross-talk between androgen and leptin signaling affects locomotor activity in female rats: Implication for polycystic ovary syndrome}

Yi Feng (1,2), Ruijin Shao (2), Birgitta Weijdegard (2), Tienpei Wang (3), Julia Johansson (2), Emil Egecioglu (2), Shan Sun (4), Hakan Billig (2), Elisabet Stener-Victorin (2)

(1) Department of Neurobiology and Integrative Medicine, Shanghai Medical College of Fudan University, Shanghai, China, (2)Department of Physiology/Endocrinology, Institute of Neuroscience and Physiology, The Sahlgrenska Academy at University of Gothenburg, Gothenburg, Sweden, (3)Department of Acupuncture and Moxibustion, Hubei College of Traditional Chinese Medicine, Wuhan, China, (4)Central Medical Lab, Eye Ear Nose and Throat Hospital, Fudan University, China

Adult female rats continuously exposed to androgens from prepuberty have reproductive and metabolic features of polycystic ovary syndrome (PCOS). This study evaluates how androgen exposure affects behavior and whether effects on behavior are mediated via androgen and leptin within the central nervous system. At 21 days of age, rats were randomly divided into two groups (control and PCOS, $\mathrm{n}=12$ /group) and implanted subcutaneously with 90-day continuousrelease pellets containing vehicle or 5a-dihydrostestosterone (DHT). Vaginal smears were obtained daily from age of week 10 to the end of the experiment. At the age of week 15, open field (locomotor, exploratory and anxiety-like behavior), elevated plus maze (anxietylike behavior) and objects recognition (non spatial memory) tests were carried out in both control (estrus) and PCOS rats. Cerebral cortex, hippocampus, amygdala and hypothalamic protein expression were measured by immunohistochemistry and western blot. PCOS rats were acyclic and displayed increased body weight and fat depots. Further, PCOS rats displayed an anxiety-related behavior in the open field test, with decreased number of peripheral part (PP) and central part $(\mathrm{CP})$ crossings and in the elevated plus-maze with decreased number of open arm (OA) and close arm (CA) entries, whereas number of grooming was increased. There were no dif- ference on non aptial memory. Hypothalamic AR protein expression and the number of AR-and leptin- immunoreactive cells were increased, as well as hippocampus leptin-immunoreactive cells in PCOS rats compared to controls. Thus, the anxiety-like behaviour in PCOS rats may be mediated by androgen and leptin activities within the central nervous system.

\section{12-4}

\section{Long-term estrogen deprivation leads to increased Alzheimer's disease-related protein induction and increased damage to the hippocampus following ischemic stroke}

Darrell Brann (1), Ruimin Wang (2), Erin Scott (1), Dong Han (1), Quan-Guang Zhang (1)

(1)Medical College of Georgia, USA, (2)North China Coal Medical University, China

Surgical menopause and menopause in humans is associated with an increased risk of Alzheimer's disease (AD) and cognitive defects, although the mechanisms are poorly understood. The current study examined the effect of long-term estradiol (E2) deprivation (10 weeks in ovariectomized rats) on the hippocampus with and without E2 replacement. Animals were subjected to 4-vessel global ischemia at the end of 1 week or 10 weeks E2 deprivation, with or without E2 replacement. The results revealed that while E2 was neuroprotective in short-term (1 week) ovariectomized animals, E2 nongenomic signaling, antioxidant action and neuroprotection in the hippocampus CA-1 region were lost after long-term E2 deprivation; and this loss was tissue-specific, as the uterus remained responsive to E2. Correspondingly, a remarkable loss of ER $\alpha$, but not ER $\beta$, was observed in the CA-1 following long-term E2 deprivation, with no change observed in the uterus. In addition, there was a dramatic increased sensitivity of the hippocampal CA-3 area to stroke damage in the long-term E2 deprived animals, and a corresponding profound increase in $\mathrm{AD}$ proteins, including $\beta$-amyloid, BACE-1, and phospho-tau. As a whole, the study provides support and mechanistic insights for a critical period of E2 replacement in the hippocampus, and demonstrates a heretofore unknown hypersensitivity of the CA-3 region to ischemic injury and $\mathrm{AD}$ protein induction after prolonged hypoestrogenicity.

\section{Nuclear receptors}

March 28 (Sun.) 11:10-12:00 Room 17

\section{13-1}

\section{Dysfunctional RORalpha signalling effects glucose} homeostasis and insulin signalling

Patrick Lau (1), Rebecca L. Fitzsimmons (1), Michael A. Pearen (1), George E.O. Muscat (1)

(1)Institute for Molecular Bioscience, The University of Queensland, St. Lucia, Queensland, Australia

Homozygous staggerer mice $(\mathrm{sg} / \mathrm{sg})$, with aberrant, and attenuated retinoic acid receptor-related orphan receptor (ROR) alpha signalling in all tissues display reduced adiposity. Moreover, this line of mice are refractory to the adverse effects of high fat diet in the context of weight gain. We began an investigation to understand whether RORalpha action was also involved in the control of carbohydrate metabolism. Homozygous $\mathrm{sg} / \mathrm{sg}$ animals exhibit display reduced blood glucose levels. Glucose (and insulin) tolerance tests, coupled to Illumina expression profiling (and bioinformatic analysis), and qPCR validation have provided insights into the role of RORalpha in the regulation of the PI3K/AKT signaling pathway and the control of glucose homeostasis. We will present data demonstrating the role of RORalpha in the modulation of insulin signaling. In summary, we propose that RORalpha is a critical regulator of skeletal muscle glucose metabolism. 
0 13-2

\section{CAG and GGN repeat lengths and CAG/GGN haplotypes variation in androgen receptor gene and prostate carcinoma in Nigeria men}

\section{Oluyemi Akinloye (1), Joerg Gromoll (2), Manuela Simoni (3)}

(1)Department of Chemical Pathology, College of Health Sciences, Ladoke Akintola University of Technology, Nigeria, (2)Centre for Reproductive Medicine and Andrology of the University of Muenster, Muenster, Germany, (3)Dept. of Medicine, Endocrinology, Metabolism and Geriatrics, University of Modena and Reggio Emilia, Via Giardini, Italy

Prostate cancer has become the number one cancer in Nigerian men and constitutes $11 \%$ of all male cancers. The growth of the prostate gland is dependent on circulating androgens and intracellular steroid signaling pathways. The effects of androgens are mediated through the androgen receptor (AR), a ligand-activated nuclear transcription factor encoded by AR gene. The AR polymorphisms; $\mathrm{CAG}$ and GGN repeats have been implicated as possible risk factor in prostate cancer. Thus far, existing supporting data are still scanty and none from sub-Saharan African population. Therefore, we have investigated the possible association between AR polymorphism repeats length (CAG and GGN) and prostate cancer in Nigerian men. A total of 261 subjects consist of 70 prostate cancer patients, 68 with benign prostate hyperplasia and 123 age matched apparently normal subjects as controls. CAG and GGN repeats length were determined by fragment length analysis using GeneScan. The CAG repeats length of prostate cancer and benign prostate hyperplasia men compared to control were significantly different $(\mathrm{p}<0.05)$ with reduce length of $\mathrm{CAG}$ repeats showing a significant odd ratio in both cases. However, this significant pattern was not observed in GGN repeat which show no significant different in percentage distribution between cases and controls ( $\mathrm{p}>0.05$ ). The different possible CAG and GGN haplotype variation shows no significant different between cases and controls ( $>0.05$ ), except that haplotype CAG-GGN 21 show a higher preponderant for controls. This study, the first from sub-Saharan Africa support the hypothesis that reduced CAG repeats length is a risk factor in prostate carcinoma.

\section{13-3}

\section{HMGA1a induces aberrant splicing of estrogen receptor $a$ in MCF-7 breast cancer cells}

\section{Kenji Ohe (1), Toshiaki Utsumi (2), Akila Mayeda (1)}

(1)Institute for Comprehensive Medical Science, Fujita Health University, Aichi, Japan, (2)Department of Breast Surgery, School of Medicine, Fujita Health University, Aichi, Japan

The well known transcription factor, HMGAla, also known as an oncogene, causes aberrant splicing of Presenilin-2 found in sporadic Alzheimer's disease, through sequence specific RNA binding. We have recently resolved the molecular mechanism, i.e., HMGAla traps U1 snRNP to the 5' splice site, leading to a defect in early spliceosome assembly. Since HMGA1 protein levels correlate with its tumorigenic malignancy, we are interested in the potential of HMGA1a as an aberrant splicing factor in cancer.

Estrogen-dependent growth of breast cancers relies on Estrogen Receptor- $\alpha(E R-\alpha)$. The N-terminal truncated isoform of Estrogen Receptor- $\alpha$ (ER- $\alpha 46)$ induces G0/G1 arrest when breast cancer cells (MCF-7) become confluent. ER- $\alpha 46$ mRNA is a result of exon 1 skipping which joins non-coding exon E1 and exon 2. We thus transiently expressed HMGA1a in MCF-7 cells and checked the expression of ER- $\alpha$ transcripts. Surprisingly HMGA1a induced exon 1 skipping and generated more ER- $\alpha 46$ mRNA, however, ER- $\alpha 46$ protein was downregulated. To confirm this event, we prepared competitor RNA fragment containing the HMGA1a-binding site of presenilin-2 with a nuclear localization signal and expressed it in MCF-7 cells. This competitor RNA would act as a decoy by binding endogenous HMGA1a, which was indeed the case, and inhibited ER- $\alpha 46$ mRNA expression while a control RNA did not. When HMGAla was overexpressed in these competitor RNA expressed cells, ER- $\alpha 46$ mRNA was re-expressed.

This would be the first case of oncogenic product HMGAla that functions as an aberrant splicing inducer in cancer.
0 13-4

RANKL upregulates mouse RANK gene transcription through the NFAT binding site of RANK gene promoter

Riko Kitazawa (1), Junko Ishii (1), Satomi Mukai (1), Takeshi Kondo (1), Kiyoshi Mori (1),

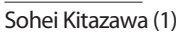

(1)Department of Pathology, Kobe University Graduate School of Medicine, Kobe, Japan

Receptor Activator of NF-KB (RANK) expressed on osteoclasts and their precursors is a receptor for RANK Ligand (RANKL). Signals transduced by RANKL-RANK interaction induce genes essential for the differentiation and function of osteoclasts partly through the direct binding of NFATc1 to target gene promoters. We have cloned a $6-\mathrm{kb}$ fragment containing the 5'-flanking region of the mouse RANK gene and found the three putative NFAT binding sites $(-370$, -550 and -720$)$. Here, we examined the regulatory mechanism of the RANK gene by RANKL signaling through NFATc1 binding. In vitro RANKL treatment of RAW cells increased RANK expression, and transfection of an NFATc1-expression vector also increased RANK and TRACP mRNA expression. Furthermore, NFATc1 knockdown by siRNA nullified the inducible effect of RANKL on RANK expression. By EMSA, an oligonucleotide (-388/-353) showed specific DNA protein binding, whereas no specific DNA protein binding was observed in nucleotides (-569/-537) and (-735/-704). Co-transfection with an NFATc1-expression vector and the promoter constructs showed that NFATc1 increased RANK promoter activity 2 -fold in RAW cells. Mutagenesis of the putative NFAT site (-370) nullified the inducible effect of NFATc1 on promoter activity; mutagenesis of sites (-550) and (-720) revealed no change in the inducible effect of NFATc1. Taken together, these results indicate that RANK transcription is positively regulated by RANKL signal through the direct binding of NFATc1 to its specific binding site of the RANK gene promoter, suggesting the presence of a positive feedback mechanism of gene expression that promotes differentiation of RANKpositive committed precursors to mature osteoclasts.

\section{Obesity and adiposcience 2}

March 29 (Mon.) 11:10-12:00

Room 11

0 14-1

\section{Gene analysis of congenital generalized lipodystrophy in} Japan

Toru Kusakabe (1), Ken Ebihara (1,2), Takeru Sakai (1), Daisuke Aotani (1), Sachiko Yamamoto-Kataoka (1), Yuji Yamamoto (1), Megumi Aizawa-Abe (1), Lichit Miyamoto (1), Kiminori Hosoda (1), Kazuwa Nakao (1)

(1)Department of Medicine and Clinical Science, Kyoto University Graduate School of Medicine, Kyoto, Japan, (2)Translational Research Center, Kyoto University Hospital, Kyoto, Japan

Congenital generalized lipodystrophy (CGL) is a rare disorder characterized by near complete absence of body fat at birth or early infancy. Diabetes mellitus accompanied by insulin resistance, hypertriglyceridemia, fatty liver, cardiomegaly, acromegaloid features and acanthosis nigricans can usually be present. CGL is frequently inherited in an autosomal recessive fashion. Seipin and AGPAT2 have been identified as causative genes for CGL. Seipin mutations were found in patients originating from Europe and the Middle East. APAT2 mutations were found predominantly in African ancestry. However, direct estimates for the prevalence rate of CGL and gene analysis of CGL are not fully performed in Japan. At first, we investigated the number of patient with lipodystrophy in Japan by inquiry survey in members of Japan Endocrine Society and found 21 CGL patients. Then, we performed gene analysis in ten CGL patients from different geographical areas of Japan. (percent body fat, $5.5 \pm 0.6$ $\%$; leptin level, $1.0 \pm 0.1 \mathrm{ng} / \mathrm{ml})($ mean $\pm \mathrm{SE}$ ). We found three different homozygous mutations in Seipin gene (Y187C, E189X and R275X) from eight CGL patients. We did not find any mutations of AGPAT2 genes in these CGL patients. Our results suggest that Seipin is a major causative gene for CGL in Japan. 
0 14-2

NAFLD with selective T2DM: An independent clinical entity?

Shen Qu (1), Qing Liu (2), Wenjun Li (1), Shengguan Chen (1), Yuqi Han (1), Jiying Wang (1), Peng Yang (1), Xiaoyun Cheng (1)

(1)Tenth People's Hospital of Tongji University , Shanghai, China, (2)Youan Hospital of Capital Medical University Peking, China

Selective T2DM exhibit a state paradoxically more severe than pure T2DM (hyperglycemia without hypertriglyceridemia). To date, there is limited data on the NAFLD patients with the sub-entity. In this study, we aim to figure out the glucose metabolic characteristics in NAFLD patients. Thirty-six NAFLD diagnosed by MRS were classified in selective T2DM, pure T2DM , hyperlipidemia and NAFLD alone. The liver fat contents increased in NAFLD with selective T2DM than T2DM $(\mathrm{P}=0.023)$, hyperlipidemia $(\mathrm{P}=0.023)$ and NAFLD alone ( $\mathrm{P}=0.00072)$. TG and TC were higher and HDL-C was lower in the NAFLD with selective T2DM group than those with pure $\mathrm{T} 2 \mathrm{DM}$ group $(\mathrm{P}=0.03$ and $\mathrm{P}=0.0006)$. Plasma glucose concentration of $120 \mathrm{~min}$ OGTT progressively increased from NAFLD with selective T2DM to T2DM $(\mathrm{P}=0.01)$, hyperlipidemia $(\mathrm{P}=0.000)$ and NAFLD alone $(\mathrm{P}=0.01)$.In response to the glucose load, NAFLD with hyperlipidemia or NAFLD alone showed significantly higher insulin levels than NAFLD with selective T2DM or T2DM .The insulin resistances were higher in subjects with hyperlipidemia and NAFLD alone than those with selective T2DM and pure T2DM, in which, NAFLD with selective T2DM group higher than those with pure T2DM ( 4.7 vs. $2.4 \mathrm{pmol} / \mathrm{min} \mathrm{P}=0.05)$. The insulin secretion did not differ between NAFLD subjects with selective T2DM or pure T2DM as compared with normal control. Diabetes with both hyperglycemia and hyperlipidemia had highest liver fat contents; Hyperlipidemia or fat liver appeared to be stronger inductor of hyperinsulinemia than plasma glucose load, in which the mechanisim of hyperinsulinemia is primarily caused by B-cell hypersecretion.

\section{14-3}

IRS-1 gene polymorphism in Indian subjects with metabolic syndromes

Surya K. Singh (1), Devi D. Pratyush (1), Ashok Kumar (1)

(1)Department of Endocrinology and Metabolism, Institute of Medical Sciences and Department of Biotechnology, Banaras Hindu University, Varanasi, India

Background: Insulin receptor substrate - 1 mediates insulin action. The previously reported polymorphism of Gly972Arg is considered a candidate gene for IRS-1 abnormality and subsequent insulin resistance. This study was designed to observe the prevalence of IRS1 gene polymorphism in association with metabolic syndrome. Material and Methods: Healthy control subjects $(\mathrm{n}=106)$ with completely normal oral glucose tolerance test and a negative family history for T2DM, hypertension and lipid abnormality along with subjects $(n=89)$ fulfilling the IDF metabolic syndrome criteria were screened for the IRS-1 G972R single nucleotide polymorphism by PCR-RFLP in North Indians. Results: A total of 385 metabolic syndrome patients and 106 controls participated in the initial screening programme. The genotype GG/GR heterozygous polymorphism frequency of IRS-1 G972R was found in $7.86 \%$ of 89 metabolic syndrome cases and $4.71 \%$ of 106 in the NGT groups. The frequency of IRS-1 gene polymorphism was not significantly different between the two groups. Conclusion: This study suggests that polymorphism of IRS1 gene has no effect on functionality of the gene product and therefore not a candidate gene for insulin resistance in our study population.
0 14-4

Metabolic syndrome (MetS) in $\mathbf{2 6 1 0}$ growth hormone deficient (GHD) patients before GH treatment start: A KIMS (Pfizer International Metabolic Database) analysis

Maria Koltowska-Haggstrom (1), Roger Abs (2), Anders F. Mattsson (1), Anton Luger (3), Maria Thunander (4), Miklos I Goth (5), Patrick Wilton (1), Johan Verhelst (6)

(1)KIMS Medical Outcomes, Pfizer Endocrine Care, Sollentuna, Sweden, (2)Department of Endocrinology, University of Antwerp, Belgium, (3)Department of Endocrinology, University of Vienna, Austria, (4) Department of Endocrinology, Kronoberg and Lund University, Vaxjo, Sweden, (5)Department of Endocrinology, University of Budapest, Hungary, (6)Department of Endocrinology, Middelheim Hospital Antwerp, Belgium

\section{OBJECTIVE}

To identify MetS and non-MetS in hypopituitary patients with adult onset GHD and to compare levels of cardiovascular (CV) risk factors.

\section{SUBJECTS AND METHODS}

MetS was defined by increased waist circumference and presence of 2 or more CV risk factors: hypertension, increased triglycerides, decreased HDL-cholesterol, and diabetes mellitus. The complementary group were defined as non-MS.1305 with MetS [51\% males, mean age $51.6(\mathrm{SD}: \pm 12.1 \mathrm{yrs})$, BMI $\left.31.2\left( \pm 5.2 \mathrm{~kg} / \mathrm{m}^{2}\right)\right]$ and 1305 non-MetS [51\% males, mean age $47.0( \pm 13.3 \mathrm{yrs})$, BMI $26.8( \pm 4.9$ $\left.\mathrm{kg} / \mathrm{m}^{2}\right)$ ] from KIMS were found. Regression analyses with adjustment for age, gender and BMI were done on relevant variables measured before GH treatment start.

RESULTS

MetS group differed unfavourably from non-MetS group in estimated GHD duration $(\mathrm{p}<0.05)$, waist-hip ratio $(\mathrm{p}<0.0001)$ and total cholesterol $(p<0.05)$. An adverse trend for waist-hip ratio, waist, HDL-cholesterol, triglycerides, and blood pressure was observed with increasing number of MetS parameters. History of cardioand cerebrovascular disorders and diabetes were more frequently encountered in the MetS group. No significant differences were found for IGF-I SDS, lean and fat mass, and LDL-cholesterol. The relative frequency of patients with MetS was similar between countries, aetiologies of GHD, and number of additional pituitary deficiencies.

\section{CONCLUSION}

MetS was found in 50\% of untreated GHD adult onset patients, who are characterized by a more adverse cardiovascular risk factor profile and a history of an increased frequency of cardiovascular complications.

\section{Tumor}

March 29 (Mon.) 11:10-12:00

\section{15-1}

Epigenetic regulation of genes involved in the intratumoral estrogen production of post-menopausal breast cancer

Kevin C. Knower (1), Sarah Q. To (1,2), Evan R. Simpson (2,3), Colin D. Clyne (1,2)

(1)Cancer Drug Discovery, Prince Henry's Institute, Clayton, Australia, (2)Department of Biochemistry and Molecular Biology, Monash University, Clayton, Australia, (3)Obesity and Metabolism, Prince Henry's Institute, Clayton, Australia.

The epithelial-stromal microenvironment of breast cancer (BC) results in dramatic gene expression changes compared to normal tissue. Despite these transcriptome changes, genetic alterations are restricted to epithelial cells, underlying the important epigenetic influence on gene regulation in the stroma. The progression of estrogen receptor positive $(\mathrm{ER}+)$ tumors in post-menopausal women is dependent on sustained intratumoral estrogen production, mediated by an increase in aromatase expression in cancer-associated fibroblasts (CAFs) surrounding ER+ tumours. This study aimed at identifying epigenetic changes between normal fibroblasts and CAFs that promote an increase in aromatase expression encoded by the gene CYP19. We show (i) that inhibition of DNA methylation in normal breast fibroblasts obtained from mastectomy with 5-aza-deoxycyti- 
dine results in a significant 40 -fold induction in CYP19 mRNA, a result also observed in breast epithelial cells; (ii) that patient ER+ status may correlate with promoter DNA hypomethylation of upstream regulators of CYP19 in CAFs, including the prostanoid receptor EP2 involved in the cAMP induction of CYP19; and (iii) that paracrine signaling pathways that up-regulate CYP19 in CAFS are epigenetically silenced in non-malignant epithelial cells. This study uncovers a new layer of complexity in the regulation of aromatase and evokes the possibility that inappropriate epigenetic regulation may give rise to a subsequent increase in aromatase levels in the breast. The use of epigenetic therapies as a selective antagonist for aromatase transcription may therefore provide future avenues in patient care.

\section{15-2}

\section{Case report of a patient with two independent well- differentiated midgut neuroendocrine tumors (WDNT) - diagnostic doubts and treatment options}

\section{Alicja B. Hubalewska-Dydejczyk (1), Anna Sowa-Staszczak (1), Agnieszka Stefanska (1), Dorota Pach (1), Piotr Szybinski (2), Andrzej Kulig (2), Romana Tomaszewska (3), Robert Chrzan (4)}

(1)Endocrinology Department, Nuclear Medicine Unit, Medical College, Jagiellonian University, Krakow, Poland, (2)Ist Department of Surgery, Jagiellonian University, Medical College, Krakow, Poland, (3) Department of Clinical and Experimental Pathomorphology, Jagiellonian University, Medical College, Krakow, Poland, (4)Department of Radiology, Jagiellonian University, Medical College, Krakow, Poland

A 43-year-old man was admitted to Surgery Department because of abdominal pain, vomiting, weight loss and flushes. CT examination revealed upper and middle abdomen tumor of about 110x110 mm. Histopathological analysis of the tissues obtained during the exploratory laparotomy confirmed WDNT. The patient received 5 doses of chemotherapy without any response. A positive result of SRS gave the possibility of PRRT. The patient was treated with the total dose of $400 \mathrm{mCi}$ of Y-90 DOTA-TATE. CT performed after the radioisotope therapy revealed regression of the tumor size to $65 \times 72 \times 94 \mathrm{~mm}$. Decrease in CgA level and release of symptoms were also observed. The patient underwent another laparotomy. During the surgery partial excision of the tumor was performed (histopathological examination - well-differentiated neuroendocrine cancer). Additionally, the changed appendix was also excised. The second focus of WDNT with Ki67 < 1\% was found in the appendix. A pathologist confirmed the above-mentioned lesions as independent. The following treatment with long-acting somatostatin analogues and $300 \mathrm{mCi}$ of Y-90 DOTA-TATE resulted in further regression of the tumor size to $35 \times 25 \mathrm{~mm}$. Consecutive laparotomy is considered. If complete tumor removal might be achieved is an open question. The above case report shows the efficacy of combined therapy with the use of "hot" and "cold" somatostatin analogues not only in controlling the symptoms of the disease but also in obtaining tumor size regression making surgical intervention possible. Due to the surgery another neuroendocrine tumor focus was found, which was not detected by the previously used diagnostic methods.

\section{5-3}

\section{Proteomic profiling of papillary thyroid carcinoma}

Yoshiyuki Ban (1), Gou Yamamoto (2), Michiya Takada (1), Yoshio Ban (3), Kazuo Shimizu (4), Haruki Akasu (4), Takehito Igarashi (4), Tetsuhiko Tachikawa (2), Tsutomu Hirano (1)

(1)Division of Diabetes, Metabolism, and Endocrinology, Department of Medicine, Showa University School of Medicine, Tokyo, Japan, (2)Department of Oral Pathology, School of Dentistry, Showa University, Tokyo, Japan, (3)Ban Thyroid Clinic, (4)Division of Endocrine Surgery, Department of Surgery, Nippon Medical School, Tokyo, Japan

Papillary thyroid carcinoma (PTC) is the most common endocrine malignancy, but most patients diagnosed with this type of thyroid cancer have an excellent prognosis. Recent studies using gene array technology have attempted to use a hypothesis-generating approach to understand thyroid neoplasms, but these studies rely on mRNA differences, which may or may not be related to significant biological processes. To better understand the basic mechanisms of tumor development and to identify potential new biomarkers, we performed LC/MS/MS analysis on pooled protein extracts from patients with PTC and compared the results with those from normal thyroid tissue. We detected 447 types of protein in PTC and 525 in normal thyroid gland. Among these proteins, 50 were specific to PTC and 128 were specific to normal thyroid gland. We also performed realtime RT-PCR using four known markers of PTC. Reproducibility was confirmed with these markers, but the new candidate marker $\mathrm{B}$ did not show large variations in expression levels. Therefore, we believe that candidate marker B should be examined in an immunohistochemical study. Our protein data obtained by LC/MS/MS analysis of thyroid gland tissue sections allowed the precise characterization of each lesion. We may have also identified an important new marker using this technique. With regard to candidate marker B identified here, it may play a role at the protein level, as significant differences were not detected at the gene expression level. Using this method, we may thus be able to identify new markers that cannot be distinguished on gene expression analysis.

\section{15-4}

\section{miRNA expression in a human papillary thyroid carcinoma} cell line varies with invasiveness

Zhongyan Shan (1), Yun Gao (1), Haixia Guan (1), Jinyuan Mao (1), Chenling Fan (1), Weiping Teng (1)

(1)Department of Endocrinology and Metabolism, the First Affiliated Hospital, China Medical University, Shenyang, P.R. China

Background Several recent studies have identified specific miRNAs that are central to metastatic progression. However, little is known about the expression of miRNAs in PTC, or about their utility as biomarkers for predicting metastatic behavior and clinical outcome. The purpose of these studies was to explore the expression pattern of miRNAs associated with invasion and metastasis of human papillary thyroid carcinoma (PTC). Methods A transwell invasion chamber was used to select progressively more invasive cancer cell populations from a clonal cell line of human PTC with cervical lymph node metastasis, IHH-4. The colony formation assays were used to detect the tumorigenicity of the selected sublines. Then metastasisrelated miRNAs differentially expressed between them were analyzed utilizing the miRNA microarray technique. Results Three sublines with progressive invasiveness, designated IHH-4M-1, IHH-4M-2 and IHH-4M-3, were obtained through this in vitro transwell invasion chamber selection process. The sublines manifested an increase in colony-forming ability on soft agar and metastatic potency in nude mice. We found that 11 metastasis-related miRNAs were differentially expressed between the invasive subpopulations and their control subpopulations by miRNA microarray analysis. Conclusions miRNA deregulation in the thyroid could be a critical component of PTC initiation and development. In this study, we obtained interesting information regarding differential expression of metastasis-related miRNAs with statistical significance. These miRNAs may significantly impact metastasis and malignant transformations. Our results indicate a need for further studies to determine the mechanisms of miRNA expression alteration and to explore how these miRNAs may contribute to tumor metastasis.

\section{Reproduction and development}

March 29 (Mon.) 11:10-12:00

Room 13

$016-1$

\section{Effect of polymorphisms in selected genes involved in pituitary-testicular function on reproductive hormones and phenotype in aging men}

Ilpo T. Huhtaniemi (1), Stephen R. Pye (2), Kate L. Holliday (2), Felipe F. Casanueva (3), Fred C.W.Wu (4)

(1)Department of Surgery \& Cancer, Division of Cancer, Reproductive Biology \& Clinical Obstetrics \& Gynaecology, Imperial College London, London, UK, (2)ARC Epidemiology Unit, The University of Manchester, Manchester Academic Health Science Centre, Manchester, UK, (3)Department of Medicine, Santiago de Compostela University, Complejo Hospitalario Universitario de Santiago, Santiago de Compostela, Spain, (4)Department of Endocrinology, The University of Manchester, Manchester Academic Health Science Centre, Manchester Royal Infirmary, Manchester, UK

Polymorphisms of genes involved in regulation, biosynthesis, metabolism and actions of testicular sex hormones may influence hormone 
balance and phenotype of aging men. This question was studied on the cross-sectional baseline data of the European Male Aging Study (EMAS), a multi-national prospective cohort observational survey of community-dwelling men. The study population consists of 2,749 men, aged 40 to 79 (mean $60.2 \pm 11.2$ [SD]) years, randomly recruited from 8 European centers. We report here the relationships between polymorphisms in genes related to pituitary-testicular endocrine function and health status. Forty-three polymorphisms were genotyped in androgen receptor (AR), estrogen receptor- $\alpha$ and $-\beta$ (ESR1 and ESR2), steroid 5a-reductase type II (SRD5A2), 17 $\alpha$ hydroxylase/17,20-lyase (CYP17A1), aromatase (CYP19A1), sex hormone-binding globulin (SHBG), luteinizing hormone $\beta$-subunit (LHB) and luteinizing hormone receptor (LHCGR). Associations between polymorphisms and endocrine, metabolic and phenotypic parameters related to aging and sex hormone action were assessed. Several polymorphisms in SHBG, ESR2, AR, CYP19A1 and $L H B$ were significantly associated with circulating levels of SHBG, LH, total, free and bioavailable testosterone and estradiol, the LH $\times$ testosterone product, and indices of insulin sensitivity. Apart from several associations between genes affecting estrogen levels and heel ultrasound parameters, no associations existed between polymorphisms and non-hormonal variables (anthropometry, blood lipids, blood pressure, hemoglobin, prostate symptoms, prostate-specific antigen, sexual dysfunction, cognition). In conclusion, in aging men, polymorphisms in genes related to the pituitary-testicular endocrine function significantly influence circulating LH, testosterone and estradiol levels, but the downstream effects may be too small to influence secondary phenotypic parameters.

\section{16-2}

\section{Identification of a novel enhancer region in the human steroidogenic acute regulatory protein (StAR) gene}

Tetsuya Mizutani (1), Takashi Yazawa (1), Miki Uesaka (1), Yoshihiko Inaoka (1), Yunfeng Ju (1), Reiko Okada (1), Kaoru Matsuura (1), Yasue Kamiki (1), Akihiro Umezawa (2), Kaoru Miyamoto (1)

(1)Department of Biochemistry, University of Fukui, Fukui, Japan, (2)National Research Institute for Child Health and Development

StAR is a cholesterol transporter from the outer to the inner mitochondrial membrane, the process of which is the rate limiting step for steroidogenesis. It is well known that Steroidogenic factor-1/ adrenal-4-binding protein (SF-1) play an essential role for the expression of human StAR gene. Transcriptional regulation of the human StAR promoter has been well characterized, but analysis of its enhancer region has not. We recently demonstrated that SF-1 induced the differentiation of bone marrow-derived mesenchymal stem cells (MSCs) into steroidogenic cells such as Leydig or adrenocortical cells. Here we show a novel SF-1 dependent transcriptional regulation of human StAR gene in the differentiated MSCs and the steroidogenic cell lines.

We identified novel SF-1 binding sites in the StAR gene about $3,000 \mathrm{bp}$ upstream from the transcriptional start site. We demonstrated by a luciferase reporter system that the upstream region was important for the activation of gene expression. Interestingly, nucleosome eviction has been found at those SF-1 binding sites (both promoter and enhancer region) by ChIP assay. Furthermore, chromosome conformation capture analysis revealed that DNA loop was formed between the promoter and the enhancer regions. These results indicate that the novel enhancer region participates in the human StAR gene activation by orchestrating chromatin loop architecture.
O 16-3

Effects of growth differentiation factor 9 (GDF9) and activin A on steroidogenic acute regulatory protein (StAR) and progesterone production in human granulosa-lutein cells

Feng-Tao Shi (1), Anthony P. Cheung (1), Christian Klausen (1), He-Feng Huang (2), Peter C.K. Leung (1)

(1)Department of Obstetrics and Gynaecology, Child and Family Research Institute, University of British Columbia, Vancouver, British Columbia, Canada, (2)Department of Obstetrics and Gynecology, Zhejiang University School of Medicine, Zhejiang, Chin

Background: We have reported that GDF9 can enhance activin A $\left(\beta_{\mathrm{A}} \beta_{\mathrm{A}}\right)$-induced inhibin $\mathrm{B}\left(\alpha \beta_{\mathrm{B}}\right)$ secretion in human granulosa-lutein (hGL) cells, but its effects on StAR, ovarian steroidogenic enzymes, and progesterone production are unknown. We undertook this study to further evaluate GDF9 in this regard.

Methods: hGL cells from women undergoing IVF treatment were cultured with and without siRNA transfection targeted at inhibin $\alpha$ subunit or GDF9 before treatment with GDF9, activin A, FSH or combinations. We compared StAR, P450scc and 3BHSD expression in hGL cells and progesterone levels in culture media. mRNA and protein levels were assessed with real-time RT-PCR, immunoblotting and ELISA as appropriate. Data were analyzed by ANOVA followed by Tukey's test.

Results: Activin A alone reduced basal and FSH-induced progesterone production by decreasing StAR expression, the rate-limiting step in steroidogenesis, but not P450scc and 3BHSD. GDF9 attenuated these activin A effects on StAR and progesterone. After transfection of $\alpha$ subunit siRNA, basal activin A levels increased $(p<0.001)$ while basal and activin A-induced inhibin B levels (with and without GDF9) decreased. Furthermore, the effects of GDF9 in reversing the suppressive effects of activin $A$ on progesterone production was attenuated $(\mathrm{p}<0.001)$. Transfection of GDF9 siRNA decreased GDF9 as expected and led to lower StAR expression and progesterone secretion than those observed with activin A treatment alone. Conclusion: GDF9 attenuates the suppressive effects of activin A on StAR expression and progesterone production by increasing the expression of inhibin B which acts as an activin A competitor.'

\section{16-4}

\section{Corticotropin releasing hormone signalling and biological} actions in trophoblast BeWo cells

Dimitris Grammatopoulos (1), Maria Delidaki (1), Mei Gu (1), Manu Vatish (1) (1)Warwick Medical School, UK

Human pregnancy is associated with increased production of corticotrophin releasing hormone $(\mathrm{CRH})$ from the placenta. $\mathrm{CRH}$, through paracrine or autocrine actions, appears to influence placenta biology and trophoblast cell grown and invasion and tissue remodeling. To characterize $\mathrm{CRH} / \mathrm{CRH}$ receptor mediated biological effects in the syncytiotrophoblast, we used the choriocarcinoma cell line BeWo, a model of trophoblast differentiation. BeWo cells were syncytialized with forskolin treatement for $24 \mathrm{~h}$. This resulted in increased intercellular fusion and increased secretion of human chorionic gonadotropin (hCG), a hormonal marker of syncytialization. Studies employing quantitative RT-PCR showed that BeWo cells syncytialization resulted in increased syncytin- 1 and -2 (by 2 and 10 fold, respectively), CRH-R1 (by 3 fold) and placental transcription factor GCMa (by 5 fold) mRNA expression. Trophoblast and syncytiotrophoblast BeWo cells expressed functional CRH-R that upon CRH activation coupled to both ERK1/2 and $\mathrm{p} 38 \mathrm{MAPK}$ in EGF-R dependent but PI3-K-independent manner. Treatment with $\mathrm{CRH}(100 \mathrm{nM} / 24 \mathrm{~h})$ alone increased syncytin-1 and GCMa mRNA levels by 1.5 and 2 fold respectively without affecting $\mathrm{hCG}$ production. However, the presence of CRH $(100 \mathrm{nM})$ during forskolin-induced syncytialization significantly augmented forskolin-effects on syncytin- 1 but not -2 and GCMa mRNA expression by 2 fold respectively, as well as hCG release by $25 \%$. These results raise the possibility that $\mathrm{CRH}$ might play an important role in trophoblast differentiation. Interestingly, CRH actions were sensitive to TLR4 activation; the presence of lipopolysaccharide (LPS) prevented CRH potentiation of forskolin actions. Therefore, in BeWo cells the innate immune system appears to be an important regulator of placental CRH biological actions. 


\section{Signal transduction / Bone}

March 29 (Mon.) 11:10-12:00

Room 16

0 17-1

\section{Prolactin regulates actin cytoskeleton through JAK2 tyrosine kinase and JAK2 downstream targets}

Maria Diakonova (1), Leah Rider (1), Jing Tao (1), Diana Suen (1)

(1)Department of Biological Sciences, University Of Toledo, Toledo, $\mathrm{OH}$, USA

We have shown that prolactin (PRL)-activated tyrosine kinase JAK2 phosphorylates serine-threonine kinase PAK1 in vivo. We have demonstrated that wild type but not kinase dead JAK2 directly phosphorylates PAK1 in cells and in an in vitro kinase assay. We identified tyrosines 153, 201 and 285 in the PAK1 molecule as sites of JAK2 tyrosyl phosphorylation. To test whether these tyrosines participate in PRL-stimulated actin reorganization, we overexpressed WT or Y3F PAK1 (tyrosines 153, 201 and 285 mutated to phenylalanines) in MCF7 cells and assayed membrane ruffling. Overexpression of WT PAK1 stimulated membrane ruffling in response to PRL compared to control cells whereas the Y3F PAK1 blocked the ruffling. We also showed that cells overexpressing PAK1/JAK2 exhibited increased cell motility compared to cells overexpressing Y3F PAK1. Next, we have identified SH2B1 $\beta$ adapter protein as a PRL-dependent JAK2 target that enhances tyrosyl phosphorylation of JAK2 in response to PRL. PRL promoted association of SH2B1 $\beta$ with JAK2 and stimulated tyrosyl phosphorylation of SH2B1 $\beta$. We have shown that $\mathrm{SH} 2 \mathrm{~B} 1 \beta$ is an actin binding protein that cross-links actin filaments and that the two actin-binding domains of SH2B1 $\beta$ (amino acids 150-200 and 615-670) are required for maximal PRL-dependent cell ruffling and for maximal cell motility. We hypothesize that PRLactivated JAK2 promotes the formation of a multiprotein complex that includes JAK2, PAK1, SH2B1 $\beta$, actin filaments and probably other actin-binding proteins. The formation of this complex results in enhancement of JAK2 activation and leads to PRL-dependent actin cytoskeleton reorganization and cell migration.

\section{17-2}

\section{The cathepsin L gene is a direct target of FOXO1 in the skeletal muscle}

Yoshihiro Yamazaki (1,2,3), Yasutomi Kamei (1), Satoshi Sugita (1), Fumiko Akaike (1), Sayaka Kanai (1), Shinji Miura (4), Yukio Hirata (3), Bruce R. Troen (5),

Tadahiro Kitamura (6), Ichizo Nishino (7), Takayoshi Suganami (1), Osamu Ezaki (4), Yoshihiro Ogawa $(1,2)$

(1)Department of Molecular Medicine and Metabolism, Tokyo Medical and Dental University, Tokyo, Japan, (2)Global Center of Excellence Program; International Research Center for Molecular Science in Tooth and Bone Diseases, Medical Research Institute, (3)Department of Clinical and Molecular Endocrinology, Tokyo Medical and Dental University, Tokyo, Japan, (4)Nutritional Science Program, National Institute of Health and Nutrition, Tokyo, Japan, (5)Department of Medicine, Miller School of Medicine, University of Miami, Miami FL, USA, (6)Metabolic Signal Research Center, Institute for Molecular and Cellular Regulation, Gunma University, Maebashi, Gunma, Japan, (7)Department of Neuromuscular Research, National Institute of Neuroscience, National Center of Neurology and Psychiatry, Tokyo, Japan

FOXO1, a forkhead-type transcription factor whose gene expression is up-regulated in the skeletal muscle during starvation, appears to be a key molecule of energy metabolism and skeletal muscle atrophy. Cathepsin L, a lysosomal proteinase whose expression is also upregulated in the skeletal muscle during starvation, is induced in transgenic mice overexpressing FOXO1 relative to wild-type littermates. In this study, we conducted in vivo and in vitro experiments focusing on FOXO1 regulation of cathepsin L expression in the skeletal muscle. During fasting and refeeding of C57BL/6 mice, cathepsin L was regulated in parallel with FOXO1, in the skeletal muscle. Fastinginduced cathepsin L expression was attenuated in transgenic mice overexpressing a dominant-negative form of FOXO1 or in skeletal muscle specific-FOXO1 knockout mice relative to respective wildtype controls. Using $\mathrm{C} 2 \mathrm{C} 12$ mouse myoblasts overexpressing a constitutively active form of FOXO1, we showed that FOXO1 induces cathepsin L expression. Moreover, we found FOXO1-binding sites in both the mouse and human cathepsin L promoters. The luciferase reporter analysis revealed that the mouse and human cathepsin $\mathrm{L}$ promoters are activated by FOXO1, which is abolished by mutations in the consensus FOXO1-binding sites. Gel mobility shift and chro- matin immunoprecipiation assays showed that FOXO1 is recruited and binds to the cathepsin L promoter. This study provides in vivo and in vitro evidence that cathepsin $\mathrm{L}$ is a direct target of FOXO1 in the skeletal muscle, thereby suggesting a role of the FOXO1/cathepsin L pathway in fasting-induced skeletal muscle metabolic change and atrophy.

\section{17-3}

Arginine residues in the loop tip and finger 2 are contributing for the Sclerostin inhibitory function in Wnt signaling

Jogeswar Gadi (1), Ajita Jami (2), Han Seok Choi (1), Su Jin Park (2), Kyoung Min Kim (1), Sung-Kil Lim (1)

(1)Division of Endocrinology and Endocrine Research Institute, Department of Internal Medicine, Yonsei University College of Medicine, Seoul, Republic of Korea, (2)Brain Korea 21 Project for medical sciences, Yonsei University College of Medicine, Seoul, Republic of Korea

Sclerostin is a secreted glycoprotein, expressed and secreted by osteocytes and acts as a negative regulator of bone formation via Wnt signaling. The inhibitory action of Sclerostin in Wnt signaling is due to the binding of Wnt co-receptor LRP5. Inhibition of Sclerostin binding to Wnt receptor can enhance the Wnt signaling makes it as a highly interesting target for diseases related to bone loss such as osteoporosis. In order to identify the key residues essential for the sclerostin function, we choose site directed mutagenesis method and substituted the important basic residues with alanine. After the expression of the pcDNA3.1/sost-mycHis (wild type) and pcDNA3.1/msost-mycHis (mutated sost) in MC3T3-E1 cells, we found that the Sclerostin expression in the cell lysate is similar in wild type and mutants, but the secretion into the culture medium was slightly enhanced in the mutants compared with the wild type Sclerostin. In order to see the function of the Sclerostin in Wnt signaling, we used the culture medium to treat the cells. These results suggest that function of the mutated protein was significantly changed with substitutions at R119A (loop), R141A and R155A (finger2) compared with the wild type. Wild type Sclerostin can inhibit the Wnt activity in MC3T3-E1 cells remarkably, where as specific mutated residues in loop tip and finger2 inhibit slightly compared with wild type Sclerostin. This suggested that the residues R119, 141 and 155 in the Sclerostin protein might be crucial for the proper function of the Sclerostin.

0 17-4

\section{Identification of SHOX gene mutations in four Chinese} patients with Leri-Weill dyschondrosteosis syndrome

Feng Ying Gong (1), Hui juan Zhu (1), Dian xi Zhang (1), Yu fei Dai (1), Hui Pan (1), Jie ying Deng (1)

(1)Department of Endocrinology, Key Laboratory of Endocrinology of Ministry of Health, Peking Union Medical College Hospital, Beijing, China

Leri-Weill dyschondrosteosis (LWD) is a dominantly inherited skeletal dysplasia characterized phenotypically by Madelung wrist deformity, mesomelia and short stature. LWD can now be defined genetically by haploinsufficiency of the SHOX (short stature homeobox-containing) gene, located in the pseudoautosomal region of the $\mathrm{X}$ and $\mathrm{Y}$ chromosomes. In this study, we collected four patients children with LWD and their parents (including 7 females and 5 males). Nine pairs of primers were designed to amplify the total six exons of SHOX gene (SHOXa and SHOXb) extracted from the patients" blood by PCR method. The results showed the total ten point mutations were indentified, which located in exon 1, exon 3 and exon 6 in these patients. Among them, there are senven novel point mutations. They are c.-648 -647 insTTG and c. $-507 \mathrm{G}>\mathrm{C}$ in exon1 which is 5"UTR, c.439.C $>$ G(Arg147Gly) in exon 3 which is coden region of SHOX gene, c. $\times 1347 . \mathrm{G}>$ A, c. $\times 2143 . \mathrm{C}>\mathrm{T}$, c.\&times $2160 . \mathrm{A}>\mathrm{T}$, and c. $\times 2170 . \mathrm{A}>\mathrm{G}$ in exon $6 \mathrm{a}$ which is $3 \&$ quotUTR. All these mutations are herited from the patients parents. Some kinds of these mutations such as c. $-686 \mathrm{G}>$ C, c. $-680 \mathrm{G}>$ T, c. $-507 \mathrm{G}>\mathrm{C}$ and c. $657 . \mathrm{G}>\mathrm{A}$ can be identified in more than two patients. The results demonstrated there are common mutations types in chinese patients with LWD. 


\section{Adrenal 2}

March 29 (Mon.) 11:10-12:00

Room 17

0 18-1

Super-selective ACTH-stimulated adrenal venous sampling; A new diagnostic method for differentiating bilateral aldosterone-producing adenomas from idiopathic hyperaldosteronism

Takashi Watanabe (1), Masao Omura (1), Kohzoh Makita (2), Seishi Matsui (3), Yoko Matsuzawa (1), Tetsuo Nishikawa (1)

(1)Department of Endocrinology \&Metabolism, Yokohama Rosai Hospital, Yokohama, Japan, (2) Department of Radiology, Tokyo Kita Social Insurance Hospital, Tokyo, Japan, (3) Department of Radiology, Yokohama Rosai Hospital, Yokohama, Japan

Background: The distinction between unilateral and bilateral disease is essential to decide how to treat patients with primary aldosteronism (PA). Adrenal venous sampling (AVS) is recommended to determine the indication of unilateral adrenalectomy; however, usually-performing AVS cannot differentiate bilateral hyperaldosteronism of bilateral aldosterone-producing adenomas (Blt-APAs) from that of idiopathic hyperaldosteronism (IHA). To solve the problem, we developed a super-selective ACTH-stimulated AVS (SS-ACTH-AVS) and we could definitely differentiate Blt-APAs from IHA. Methods: We performed SS-ACTH-AVS using a 2.2Fr strip-tip type micro-catheter in 50 patients with PA. We sampled adrenal effluents at central and more than one tributary adrenal vein of each adrenal gland after ACTH stimulation and measured concentrations of aldosterone and cortisol. Results: In 20 patients with IHA, concentrations of aldosterone in effluents sampled at all central adrenal vein and tributaries were more than $1400 \mathrm{ng} / \mathrm{dl}$. On the other hand, in 4 patients with Blt-APAs, concentrations of aldosterone were increased more than $1400 \mathrm{ng} / \mathrm{dl}$ in effluents sampled at bilateral central veins and one tributary of each adrenal gland. Three patients diagnosed as Blt-APAs by SS-ACTH-AVS underwent unilateral semi-total adrenalectomy of affected adrenal glands containing adrenal masses and APA was diagnosed by pathological examinations of resected adrenal glands. Conclusion: We can differentiate bilateral Blt-APAs from IHA by performing SS-ACTH-AVS. We should perform a new method of SS-ACTH-AVS in some cases of bilateral hyperaldosteronism with adrenal nodule(s).

\section{18-2}

Problems in diagnostics of primary aldosteronism: Analysis of the own data

Janusz Mysliwiec (1), Lukasz Zukowski (1), Anna Grodzka (1), Agata Pilaszewicz (1), Szymon Dragowski (1), Beata Piekut (1), Agnieszka Nikolajuk (1), Maria Gorska (1)

(1)Department of Endocrinology, Diabetology and Internal Diseases, Medical University of Bialystok, Bialystok, Poland

Background: In the period of last several years increasing evidence suggest that primary aldosteronism is causing over $10 \%$ arterial hypertension. There are no gold standard methods for primary aldosteronism screening The aim of study was plasma renin activity (PRA), plasma aldosterone concentration (PAC), aldosterone to renin ratio (ARR) assessment as criteria for diagnosis of primary aldosteronism and their usefulness in clinical practice.Material and methods: 81 consecutive patients admitted to diagnose primary aldosteronism: 51 women and 30 men aged 31-69 ys. In each patient PAC and PRA were evaluated in radioimmunoassay. In 65 patients urine concentration of catecholamine metabolits was assayed and in 51 diagnostics for hypercortisolemia was done. In patients with adrenal incidentaloma the 16 row computer tomography was performed. Results: The ratio of patients with PAC over $150 \mathrm{pg} / \mathrm{ml}$ was $35 \%(\mathrm{n}=28)$. Rate of the patients with PRA under $0,07 \mathrm{ng} / \mathrm{ml} / \mathrm{h}$ was $19(\mathrm{n}=15)$. The ratio of patients whose values of ARR exceeded over 20,30,40, 50 and 180 were 55, 47, 37, 28 and $15 \%$ respectively. Conclusions: The most common indication for primary screening was presence of incidentally found adrenal mass. The quotient of plasma aldosterone concentration/ plasma renin activity at whichever cut-off point is not effective enough to select patients for further diagnostics.
0 18-3

Negative regulation of CYP11B2 expression and aldosterone production in adrenocortical carcinoma H295R cells by PPARY

Akira Uruno (1,2), Ken Matsuda (3), Naoya Noguchi (2), Takeo Yoshikawa (2), Masataka Kudo (3), Fumitoshi Satoh (3), William E. Rainey (4), Sadayoshi Ito (3), Hiroshi Okamoto (2), Akira Sugawara (1,2)

(1)Department of Biochemistry, Tohoku University Graduate School of Medicine, Japan, (2)Department of Advanced Biological Sciences for Regeneration, Tohoku University Graduate School of Medicine, Sendai, Japan, (3)Division of Nephrology, Endocrinology and Vascular Medicine, Tohoku University Graduate School of Medicine, Sendai, Japan, (4)Department of Physiology, Medical College of Georgia, Augusta, GA, USA

Introduction: PPAR $\gamma$ is a nuclear receptor for anti-diabetic agent thiazolidinedione, which exerts various physiological activities independent of lowering blood glucose. However, the role of PPAR $\gamma$ in aldosterone production has not been clarified. We thus examined the effect of PPAR $\gamma$ on CYP11B2 expression and aldosterone production. Methods: Human adrenocortical carcinoma H295R cells were transfected with $C Y P 11 B 2$ promoter reporter vectors and their luciferase activity was thereafter measured. CYP11B2 mRNA expression levels were detected by real-time RT-PCR. Aldosterone secretion from H295R cells into the media was determined by ELISA. Results: PPAR $\gamma$ overexpression attenuated angiotensin II (Ang II)-induced CYP11B2 promoter activity. Pioglitazone (Pio) suppressed Ang II- and potassium-induced CYP11B2 promoter activity, its mRNA expression, and Aldo secretion. The CYP11B2 promoter activity suppression mediated by Pio was attenuated in the presence of a PPAR $\gamma$ AF-2 domain mutant. Rosiglitazone and GW1929 also suppressed $C Y P 11 B 2$ promoter activity and aldosterone secretion. Mutation analysis revealed that Ad1/CRE element in CYP11B2 promoter was responsible for the promoter activity suppression. $\mathrm{Ca}^{2+} /$ calmodulin-dependent kinase (CaMK) inhibitor KN-93 attenuated suppression of $C Y P 11 B 2$ promoter activity, and Pio inhibited induction of $C Y P 11 B 2$ promoter activity in response to a truncated constitutively active CaMK-I (CaMK-I-295). Pio did not suppress dibutyl cyclic AMP-induced CYP11B2 promoter activity. Conclusion: PPAR $\gamma$ suppressed CYP11B2 expression and aldosterone secretion.

0 18-4

The physiological role of prolactin secretion in response to stress on adrenal hormone secretion

Sukanya Jaroenporn (1,2), Kentaro Nagaoka (3), Ryo Ohta (4), Gen Watanabe $(2,5)$, Kazuyoshi Taya $(2,5)$

(1)Primate Research Unit, Department of Biology, Faculty of Science, Chulalongkorn University, Bangkok, Thailand, (2)Laboratory of Veterinary Physiology, Department of Veterinary Medicine, Faculty of Agriculture, Tokyo University of Agriculture and Technology, Tokyo, Japan, (3) Laboratory of Animal Breeding and Reproduction, The University of Tokyo, Japan, (4)Safety Testing Laboratory, Research Administration, Hatano Research Institute, Food and Drug Safety Center, Hatano, Kanagawa, Japan, (5)Department of Basic Veterinary Science, The United School of Veterinary Sciences, Gifu University, Gifu, Japan

Prolactin (PRL) has been proposed to directly stimulate corticosterone release. However, the physiological role of PRL secretion in response to stress is rather elusive. The aim of this study was to provide results that support the hypothesis that PRL plays a role as anti-stress mediators and to highlight its effects on adrenal gland. By using in vitro cell culture system in male rats, the results showed that PRL could act directly on the adrenal gland to drive adrenal hormones secretion and increase the expression of StAR, CYP11A1 and PRLR-L mRNA. However, the effects of PRL on adrenal function were reduced by AG490, specific Janus kinase2 (Jak2) inhibitor, treatment. This finding emphasizes that PRL stimulation of adrenal hormones release is mediated through Jak2 activity. Next, we attempted to assess whether stress-induced PRL secretion is associated with enhances the secretion of adrenal hormones in response to stress. It is well known that PRL signaling through its receptor involves the Jak2/signal transducers and activators of transcription (Jak2/STAT5) pathway. Therefore, STAT5 phosphorylation was used as the indicator of PRL signaling. It is interesting to note that enhancement of STAT5 activation by restraint stress was observed. More importantly, CB-154-induced hypoprolactinemia could attenuate stress-induced STAT5 phosphorylation in adrenal gland. These results clearly demonstrate that PRL levels increase in response to stress, and it acts on the adrenal cortex to regulate adrenocortical function through Jak2/STAT5 pathway. supported by a Grant- 
in-Aid for Scientific Research(B-18310044)from the Japan Society and Grant for Development of New Faculty Staff, Chulalongkorn University.

\section{Peptides/Regeneration}

March 30 (Tue.) 10:05-10:55

Room 17

0 19-1

\section{Secretopeptidome mining for novel biologically active} peptides

Tsukasa Osaki (1), Kazuki Sasaki (1), Naoto Minamino (1)

(1)Department of Pharmacology, National Cardiovascular Center Research Institute, Osaka, Japan

Peptide hormones, neuropeptides and antimicrobial peptides represent a paradigm in which peptides cleaved from precursor proteins gain functions not ascribed to the original proteins. In previous work, peptidomic profiling studies of the naturally cleaved peptides have met with technical difficulty in determining precursor processing sites because the processed peptides subsequently undergo $\mathrm{N}$ - or $\mathrm{C}$-terminal trimming by numerous proteases. We took advantage of the fact that in neurons and endocrine cells most bioactive peptides are stored in secretory granules and released by exocytosis. To prevent the proteolytic degradation, we analyzed secretopeptidome, the entire peptide complement released by a brief exocytotic stimulus from cultured endocrine cells. In this study, we used a thyroid cell line of $\mathrm{C}$ cell origin that stores peptide hormones in secretory granules. We found that over $95 \%$ of identified peptides were derived from precursor proteins processed in secretory granules, including nine peptide hormone and neuropeptide precursors, seven graninlike proteins and three processing enzymes. In total, over $93 \%$ of the identified peptides retained initial cleavage sites recognized by processing enzymes operating in secretory granules. These results suggest that this secretopeptidomic approach is promising to discover novel bioactive peptides. However, the sequence of a given peptide does not offer information about biological activity. Since most antimicrobial peptides are positively charged, we are now conducting peptide profiling of highly basic peptides secreted from cultured cells to discover novel antimicrobial peptides.

\section{$019-2$}

\section{Regulation and roles of urocortins in human adrenal H295R cells}

\section{Kazunori Kageyama (1), Komaki Hanada (1), Toshihiro Suda (1)}

(1)Department of Endocrinology and Metabolism, Hirosaki University Graduate School of Medicine, Japan

Three urocortins (Ucns) are known as members of the corticotropin-releasing factor (CRF) family of peptides and serve as natural ligands for CRF receptors. Ucn1 and Ucn3 have potent effects on the adrenal system via the CRF receptors. The objective of the study was to explore the regulation and roles of Ucns in the adrenal system using human adrenal carcinoma H295R cells. H295R cells express Ucn1, Ucn2, Ucn3, CRF receptor type 1 (CRF1 receptor), and CRF receptor type 2a (CRF2a receptor) mRNA. Forskolin, which stimulates adenylate cyclase, and then increases intracellular cAMP production, was shown to transiently decrease Ucn1 and Ucn2 mRNA levels, but increase Ucns 1-3 mRNA levels in H295R cells. Ucn1 increased steroidogenic acute regulatory protein mRNA levels, and both cortisol and aldosterone secretion. Cell viability was reduced by both Ucn 1 and Ucn 3 via the CRF2 receptor in H295R cells. Ucn 1 and Ucn3 increased the expression of the cAMP-response element binding protein and extracellular signal-related kinases (ERK) phosphorylation. The ERK and protein kinase A pathways were involved in Ucn3-decreased cell viability.
0 19-3

GHS receptor is involved in insulin action in mouse skeletal
muscle

Keiichi Koshinaka (1), Koji Toshinai (1), Hideki Yamaguchi (1), Masamitsu Nakazato (1) (1)Division of Neurology, Respirology, Endocrinology, and Metabolism, Department of Internal Medicine, Faculty of Medicine, University of Miyazaki, Japan

Ghrelin is an endogenous ligand for growth hormone secretagogue (GHS) receptor (GHS-R). Although multiple functions of ghrelin are known in various tissues, physiological role of ghrelin/GHS-R is not yet defined. Because GHS binding sites are present in insulin-sensitive human peripheral tissues including liver, fat and skeletal muscle, we expected that endogenous ghrelin/GHS-R have a role in regulating insulin action. To identify the physiological role of GHS-R, we analyzed phenotype of GHS-R KO mice. Compared to wild-type mice, no difference was observed in body weight or skeletal muscle mass. However, epididymal fat mass in KO mice was lower than that in wild-type mice. Growth hormone and glucose levels were not different between groups, whereas insulin level in $\mathrm{KO}$ mice was lower than that in wild-type mice, suggesting that GHS-R KO mice have a higher insulin action. To test this hypothesis, mice were undertaken insulin tolerance test. Reduction of glucose in response to insulin was larger in $\mathrm{KO}$ mice than wild-type mice, showing enhanced wholebody insulin action in $\mathrm{KO}$ mice. Next we measured insulin action in skeletal muscles. When isolated skeletal muscles were incubated with insulin, 2-deoxy glucose uptake in muscles from $\mathrm{KO}$ mice was higher than muscles from wild-type mice. Glucose transporter 4 protein content and mitochondria content determined by citrate synthase activity were not different between groups. These results indicate that the GHS-R is involved in regulatory mechanisms of insulin action in mouse skeletal muscle.

\section{19-4}

\section{Tracing phenotypic reversibility of pancreatic beta-cells in vitro}

Kohtaro Minami (1,2), Kazumasa Miyawaki (1), Susumu Seino $(1,3,4)$

(1)Division of Cellular and Molecular Medicine, Kobe University Graduate School of Medicine, Japan, (2) Laboratory for Somatic Stem Cell Therapy, Foundation for Biomedical Research and Innovation, (3)Division of Diabetes, Metabolism and Endocrinology, Department of Internal Medicine, Kobe University Graduate School of Medicine, Japan, (4)Core Research for Evolutional Science and Technology (CREST), Japan Science and Technology Corp., Japan

Studies have suggested that pancreatic beta-cells undergo dedifferentiation during proliferation in vitro. However, due to limitations of the methodology, the question remains whether or not the dedifferentiated cells can redifferentiate into beta-cells. In this study, we established a novel cell tracing method for imaging insulin expression in living cells that reveals phenotypic reversibility of pancreatic beta-cells. Mice expressing GFP under control of mouse insulin 1 promoter (MIP-GFP mice) were crossbred with mice expressing monomeric red fluorescent protein (mRFP) in all cells (CAG-mRFP mice), resulting in generation of pancreatic beta-cells labeled with both green and red fluorescent proteins. The GFP-expressing betacells were obtained by using a fluorescence-activated cell sorter. When cultured with fetal mouse pancreatic cells, the sorted betacells lost GFP expression but retained mRFP expression. The cells also lost expressions of genes characteristic of the beta-cell phenotype, such as Pdx1 and glucokinase, indicating dedifferentiation. More than $30 \%$ of such dedifferentiated pancreatic beta-cells were detected in $\mathrm{S}$ or $\mathrm{G} 2 / \mathrm{M}$ phase. We found that the dedifferentiated cells can redifferentiate into insulin-expressing cells upon cultivation with a MEK1/2 inhibitor. These data provide direct evidence that adult mouse pancreatic beta-cells have potential not only for dedifferentiation but also for redifferentiation in vitro. Our method represents a useful approach to investigate phenotypic reversibility of pancreatic beta-cells. 
020

\section{Obesity and adiposcience 3}

March 30 (Tue.) 10:55-11:45

Room 17

0 20-1

PTP1B- or SHP2-deficiency in POMC neurons reciprocally regulates energy balance and glucose homeostasis

Ryoichi Banno (1,2), Derek Zimmer (2), Marybless Atienza (2), Kimberly Rak (2), Wentian Yang (2), Kendra K. Bence (2)

(1)Department of Endocrinology and Diabetes, Nagoya University Graduate School of Medicine, Nagoya, Japan, (2)Department of Animal Biology, School of Veterinary Medicine, University of Pennsylvania, Philadelphia, Pennsylvania, USA

PTP1B and SHP2 are ubiquitously expressed protein tyrosine phosphatases which have both been shown to regulate metabolism via the CNS in vivo, but the specific neurons mediating these effect are unknown. Here we show that POMC neuron-specific deletion of PTP1B (Ptpn1loxP/loxP POMC-Cre) or SHP2 ( Ptpn11loxP/loxP POMC-Cre) in mice results in reciprocal effects on weight gain and fatty liver induced by a high fat diet, leptin sensitivity, and glucose homeostasis. High-fat fed POMC-PTP1B-/- mice have reduced adiposity, lowered serum leptin levels, improved leptin sensitivity, and increased energy expenditure, whereas POMC-SHP2-/- mice have elevated body weight and adiposity on a high-fat diet, decreased leptin sensitivity, and decreased energy expenditure. POMC-PTP1B-/mice show significantly improved insulin sensitivity and glucose tolerance on a high fat diet, and hyperinsulinemic-euglycemic clamp studies reveal that insulin sensitivity is also improved on a chow diet in the absence of any weight difference. On the other hand, SHP2deficiency in POMC neurons results in elevated serum insulin levels, and impaired insulin sensitivity and glucose tolerance. These studies implicate PTP1B and SHP2 as important components of POMCneuron regulation of energy balance and glucose homeostasis.

\section{20-2}

\section{Melanocortin $3 / 4$ receptor is essential to the intracellular} signaling pathway of nesfatin-1

Emi Ishida (1), Koshi Hashimoto (1), Tetsurou satoh (1), Shuichi Okada (1), Masanobu Yamada (1), Hiroyuki Shimizu (1), Masatomo Mori (1)

(1)Department of Medicine and Molecular Science, Gunma University Graduate School of Medicine, Gunma, Japan

Nesfatin-1 is a novel anorexic peptide that reduces the appetite of rats by intraventricular and intraperitoneal administration. The anorexic effect is diminished by antagonists of Melanocortin 3/4 receptor $(\mathrm{MC} 3 / 4 \mathrm{R})$. We have recently revealed that the thirty amino residues of nesfatin-1, referred to $\mathrm{M} 30$, increased the phosphorylation level of cAMP response element binding protein (CREB) in neuroblastoma cell lines of mice. In the current study, we examined whether MC3/4R signaling pathway is involved to the phosphorylation of CREB by M30. We hypothesized either the specific signaling pathway via MC3/4R or MC3/4R gene expression per se would be essential to the nesfatin-1 signal transduction. For the former purpose, we treated the neuroblastoma cells with MC3/4R antagonist (SHU9119) or agonist (Melanotan II, MTII) followed by Western blotting analysis to detect phosphorylated CREB. For the latter one, small interfering RNA(siRNA) specific for MC4R was employed to knock down the MC4R gene expression. Furthermore, we hypothesized nesfatin- 1 would modulate $\mathrm{MC} 3 / 4 \mathrm{R}$ gene expression. For this purpose, we treated the neuroblastoma cells with M30 and performed the real-time PCR analysis to evaluate mRNA levels of MC3/4R. As a result, both M30 and MTII increased phosphorylated CREB. On the other hand, SHU9119 treatment deteriorated CREB phosphorylation induced by M30. Knockdown of 30-60\% MC4R gene expression also reduced the phosphorylated CREB by M30. Interestingly, M30 treatment significantly decreased the mRNA level of MC3/4R. From these results, we concluded that MC3/4R gene expression and the signaling pathway are essential to phosphorylate CREB by M30, and $\mathrm{M} 30$ regulates $\mathrm{MC} 3 / 4 \mathrm{R}$ gene expression.
0 20-3

Nesfatin-1-regulated oxytocinergic signaling in the paraventricular nucleus causes anorexia via melanocortin pathway

Yuko Maejima (1), Udval Sedbazar (1), Shigetomo Suyama (1), Daisuke Kohno (1), Tatsushi Onaka (2), Masato Koike (3), Yasuo Uchiyama (3), Tamas L. Horvath (4), Marcelo O. Dietrich (4), Masatomo Mori (5), Toshihiko Yada (1)

(1)Division of Integrative Physiology, Department of Physiology, Jichi Medical University School of Medicine, Japan, (2)Division of Brain and Neurophysiology, Department of Physiology, Jichi Medical University School of Medicine, Japan, (3)Department of Cell Biology and Neurosciences, Juntendo University Graduate School of Medicine, Japan, (4)Program on Cell- and Neurobiology of Energy Metabolism, Section of Comparative Medicine, Yale University School of Medicine, USA, (5)Department of Medicine and Molecular Science, Gunma University Graduate School of Medicine, Japan

Nesfatin-1 is a recently discovered anorectic peptide derived from nucleobindin2. Nesfatin-1 is localized in several brain areas including the paraventricular nucleus (PVN). However, anorectic neural pathway of nesfatin-1 remains unknown. The purpose of this study is to determine the mechanism of feeding regulation by nesfatin-1. Here we show that central injection of nesfatin-1 activated several hypothalamus nuclei including the PVN and the brain stem nucleus tractus solitarius (NTS). Intra-PVN injection of nesfatin-1 induced anorexia. This suggested that PVN was one of the target sites of nesfatin-1-induced anorexia. In the PVN, nesfatin-1 increased intercellular $\mathrm{Ca}^{2+}$ concentration $\left(\left[\mathrm{Ca}^{2+}\right]\right.$.) in single oxytocin, nesfatin-1 and oxytocin/nesfatin-1 double positive neurons. In the experiment using PVN slices, nesfatin-1 stimulated oxytocin release. Immunoelectron micrographs revealed nesfatin-1 specifically in the secretory vesicles of PVN neurons, and immuno-neutralization against endogenous nesfatin-1 suppressed oxytocin release in the PVN. These results suggested the paracrine/autocrine actions of nesfatin-1 in the PVN. Nesfatin-1-induced anorexia was abolished by an oxytocin receptor antagonist. In addition, oxytocin-induced anorexia was abolished by SHU9119, a melanocortin 3/4 receptor (MC3/4R) antagonist, suggesting that MC3/4R is involved in the downstream of nesfatin-1-regulated oxytocin neurons. Moreover, oxytocin terminals were closely associated with proopiomelanocortin (POMC) neurons in the NTS, and oxytocin increased $\left[\mathrm{Ca}^{2+}\right]$ in single POMC neurons in the NTS. In conclusion, nesfatin- 1 activates the activity and secretion of oxytocin neurons in the PVN, and oxytocin activates POMC neurons in the NTS. This circuit serves as the neural pathway for the anorectic effect of nesfatin-1.

\section{$020-4$}

\section{Induction of hypothalamic Sirt1 leads to cessation of feeding} via AgRP

Tsutomu Sasaki (1), Hye-Jin Kim (1), Masaki Kobayashi (1), Yukari-ldo Kitamura (1), Hiromi Yokota-Hashimoto (1), Tetsuya Shiuchi (2), Yasuhiko Minokoshi (2), Tadahiro Kitamura (1)

(1)Laboratory of Metabolic Signal, Institute for Molecular and Cellular Regulation, Gunma University, Gunma, Japan, (2)Division of Endocrinology and Metabolism, National Institute for Physiological Sciences, Aichi, Japan

SIR2 is an $\mathrm{NAD}^{+}$-dependent deacetylase implicated in the regulation of lifespan in species as diverse as yeast, worms, and flies. Mammalian Sirt1 is the most closely related homolog of the SIR2 gene. Pharmacological activators of Sirt1 have been reported to increase the lifespan and improve the health of mice fed a high-fat diet and to reverse diabetes in rodents. Sirtl links the energy availability status with cellular metabolism in peripheral organs including liver, pancreas, muscle, and white adipose tissue. Insulin and leptin signaling regulate food intake by controlling the expression of orexigenic and anorexigenic neuropeptides in the arcuate nucleus of the hypothalamus via Foxo1 and Stat3. Sirt1 has been reported to improve insulin sensitivity in vitro, but the role of hypothalamic Sirt1 in regulating food intake has not been addressed. We found that hypothalamic Sirt1 protein levels increase upon feeding and that Sirt1 protein turnover is regulated by the proteasome and ubiquitination in a hypothalamic cell line. Forced expression of wild-type Sirt1 in the mediobasal hypothalamus by adenovirus microinjection suppressed Foxo1-induced hyperphagia. Moreover, Sirt1 suppressed Foxo1-dependent expression of the orexigenic neuropeptide AgRP in vitro. We propose that upon feeding, Sirt1 protein is stabilized in the hypothalamus, leading to decreased Foxo1-dependent expression of orexigenic neuropeptide AgRP and cessation of feeding. 